\title{
Second order asymptotics in fixed-length source coding and intrinsic randomness
}

\author{
Masahito Hayashi
}

\begin{abstract}
Second order asymptotics of fixed-length source coding and intrinsic randomness is discussed with a constant error constraint. There was a difference between optimal rates of fixed-length source coding and intrinsic randomness, which never occurred in the first order asymptotics. In addition, the relation between uniform distribution and compressed data is discussed based on this fact. These results are valid for general information sources as well as independent and identical distributions. A universal code attaining the second order optimal rate is also constructed.
\end{abstract}

Index Terms-Second order asymptotics, Fixed-length source coding, Intrinsic randomness, Information spectrum, Folklore for source coding

\section{INTRODUCTION}

$\mathbf{M}$ ANY researchers believe that any sufficiently compressed data approaches a uniform random number. This conjecture is called Folklore for source coding (Han [1]). The main reason for this conjecture seems to be the fact that the optimal limits of both rates coincide with the entropy rate: that is, the optimal compression length equals the optimal length of intrinsic randomness (uniform random number generation) in the asymptotic first order. There is, however, no research comparing them in the asymptotic second order even though some researchers treat the second order asymptotics for variable-length source coding [2], [3]. In this paper, taking account of the asymptotic second order, we compare them in the case of the general information source in the fixed-length setting. Especially, we show by application to the case of the independent and identical distribution (i.i.d.), that the size of compression is greater than the one of intrinsic randomness with respect to the asymptotic second order. This fact implies that data generated by the fixed-length source coding is not a uniform random number.

Details of the above discussion are as follows. The size of generated data is one of the main points in data compression and intrinsic randomness. In the asymptotic setting, by approximating the size $M_{n}$ as $M_{n} \cong e^{n a}$, we usually focus on the exponential component (exponent) $a$. Smaller size is better in data compression, but larger size is better in intrinsic randomness. Both optimal exponents $a$ coincide. However, as will be shown in this paper, the size $M_{n}$ can be approximated as $M_{n} \cong e^{n a+\sqrt{n} b}$. In this paper, we call the issue concerning the coefficient $a$ of $n$ the first order asymptotics, and the issue concerning the coefficient $b$ of $\sqrt{n}$ the second order asymptotics. When the information source is

M. Hayashi is with Quantum Computation and Information Project, ERATO, JST, 5-28-3, Hongo, Bunkyo-ku, Tokyo, 113-0033, Japan. (e-mail: masahito@qci.jst.go.jp) the independent and identical distribution $P^{n}$ of a probability distribution $P$, the optimal first coefficient is the entropy $H(P)$ in both settings. In this paper, we treat the optimization of the second coefficient $b$ for general information sources. In particular, we treat intrinsic randomness by using half of the variational distance. These two coefficients do not coincide with each other in many cases. In particular, these optimal second order coefficients depend on the allowable error even in the i.i.d. case. (Conversely, it is known that these optimal first order coefficients are independent of the allowable error in the i.i.d. case when the allowable error is constant.) If the allowable error is less than $1 / 2$, the optimal second order coefficient for source coding is strictly larger than the one for intrinsic randomness. As a consequence, when the constraint error for source coding is sufficiently small, the compressed random number is different from the uniform random number. Hence, there exists a trade-off relation between the error of compression and the error of intrinsic randomness.

However, Han [1], [4], [5] showed that the compressed data achieving the optimal rate is 'almost' uniform random at least in the i.i.d. case in the fixed-length compression. Visweswariah et al.[6] and Han \& Uchida [7] also treated a similar problem in the variable-length setting. One may think that Han's result contradicts our result, but there is no contradiction. This is because Han's error criterion between the obtained distribution and the true uniform distribution is based on normalized KL-divergence (30), and is not as restrictive as our criterion. Thus, the distribution of the compressed data may not be different from the uniform distribution under our criterion even if it is 'almost' the uniform distribution under his criterion. Indeed, Han [5] has already stated in his conclusion that if we adopt the variational distance, the compressed data is different from the uniform random number in the case of the stationary ergodic source. However, in this paper, using the results of second order asymptotics, we succeeded in deriving the tight trade-off relation between the variational distance from the uniform distribution and decoding error probability of the fixed-length compression in the asymptotic setting. Further, when we adopt KL-divergence divided by $\sqrt{n}$ instead of normalized KL-divergence, the compressed data is different from the uniform random number. Hence, the speed of convergence of normalized KL-divergence to 0 is essential.

In this paper, we use the information spectrum method mainly formulated by Han[4]. We treat the general information source, which is the general sequence $\left\{p_{n}\right\}$ of probability distributions without structure. This method enables us to characterize the asymptotic performance only with the random variable $\frac{1}{n} \log p_{n}$ (the logarithm of likelihood) without any 
further assumption. In order to treat the i.i.d. case based on the above general result, it is sufficient to calculate the asymptotic behavior of the random variable $\frac{1}{n} \log p_{n}$. Moreover, the information spectrum method leads us to treat the second order asymptotics in a manner parallel to the first order asymptotics, whose large part is known. That is, if we can suitably formulate theorems in the second order asymptotics and establish an appropriate relation between the first order asymptotics and the second order asymptotics, we can easily extend proofs concerning the first order asymptotics to those of the second order asymptotics. This is because the technique used in the information spectrum method is quite universal. Thus, the discussion of the first order asymptotics plays an important role in our proof of some important theorems in the second order asymptotics. Therefore, we give proofs of some theorems in the first order asymptotics even though they are known. This treatment produces short proofs of main theorems for the second order asymptotics with reference to the corresponding proofs on the first order asymptotics.

While we referred the i.i.d. case in the above discussion, the Markovian case also has a similar asymptotic structure. That is, the limiting distribution of the logarithm of likelihood is equal to normal distribution. Hence, we have the same conclusion concerning Folklore for source coding in the Markovian case. Moreover, we construct a fixed-length source code that attains the optimal rate up to the second order asymptotics, i.e., a universal fixed-length source code. We also prove the existence of a similar universal operation for intrinsic randomness. Further, in Section VI-A, we derived the optimal generation rate of intrinsic randomness under the constant constraint concerning the normalized KL-divergence, which was mentioned as an open problem in Han's textbook[4].

Finally, we should remark that the second order asymptotics correspond to the central limit theorem in the i.i.d. case while the first order asymptotics corresponds to the law of large numbers. But, in statistics, the first order asymptotics corresponds to the central limit theorem. Concerning variablelength source coding, the second order asymptotics corresponds to the central limit theorem, but its order is $\log n$. As seen in sections VIII and $\mathrm{X}-\mathrm{K}$ the application of this theorem to variable- and fixed-length source coding is different.

This paper is organized as follows. We explain some notations for the information spectrum method in the first and the second order asymptotics in section II. We treat the first order asymptotics of fixed-length source coding and intrinsic randomness based on variational distance in section III, some of which are known. For the comparisons with several preceding results, we treat several versions of the optimal rate in this section. The second order asymptotics in both settings are discussed as the main result in section IV. We discuss the relation between the second order asymptotics and Folklore for source coding based on variational distance in section $\mathrm{V}$. In addition, we discuss intrinsic randomness based on KLdivergence, and the relation between Han[4]'s criterion and the second order asymptotics in section VI. For comparison with Han[4]'s result, we treat intrinsic randomness under another KL-divergence criterion, in which the input distributions of KL-divergence are exchanged. In section VII, the Markovian case is discussed. A universal fixed-length source code and a universal operation for intrinsic randomness are treated in section VIII. All proofs are given in section IX.

\section{NOTATIONS OF INFORMATION SPECTRUM}

In this paper, we treat general information source. Through this treatment, we can understand the essential properties of problems discussed in this paper. First, we focus on a sequence of probability spaces $\left\{\Omega_{n}\right\}_{n=1}^{\infty}$ and a sequence of probability distributions $\bar{p} \stackrel{\text { def }}{=}\left\{p_{n}\right\}_{n=1}^{\infty}$ on them. The asymptotic behavior of the the logarithm of likelihood can be characterized by the following known quantities

$$
\begin{aligned}
& \underline{H}(\epsilon \mid \bar{p}) \stackrel{\text { def }}{=} \inf _{a}\left\{a \mid \varlimsup \overline{\lim } p_{n}\left\{-\frac{1}{n} \log p_{n}(\omega)<a\right\} \geq \epsilon\right\} \\
& =\sup _{a}\left\{a \mid \varlimsup \lim p_{n}\left\{-\frac{1}{n} \log p_{n}(\omega)<a\right\}<\epsilon\right\}, \\
& \bar{H}(\epsilon \mid \bar{p}) \stackrel{\text { def }}{=} \inf _{a}\left\{a \mid \underline{\lim } p_{n}\left\{-\frac{1}{n} \log p_{n}(\omega)<a\right\} \geq \epsilon\right\} \\
& =\sup _{a}\left\{a \mid \underline{\lim } p_{n}\left\{-\frac{1}{n} \log p_{n}(\omega)<a\right\}<\epsilon\right\},
\end{aligned}
$$

for $0<\epsilon \leq 1$, and

$$
\begin{array}{r}
\left.\underline{H}_{+}(\epsilon \mid \bar{p}) \stackrel{\stackrel{\text { def }}{=} \inf _{a}\{a \mid \varlimsup}{\varlimsup} p_{n}\left\{-\frac{1}{n} \log p_{n}(\omega)<a\right\}>\epsilon\right\} \\
=\sup _{a}\left\{a \mid \varlimsup \bar{l} p_{n}\left\{-\frac{1}{n} \log p_{n}(\omega)<a\right\} \leq \epsilon\right\}, \\
\bar{H}_{+}(\epsilon \mid \bar{p}) \stackrel{\text { def }}{=} \inf _{a}\left\{a \mid \underline{\lim } p_{n}\left\{-\frac{1}{n} \log p_{n}(\omega)<a\right\}>\epsilon\right\} \\
=\sup _{a}\left\{a \mid \underline{\lim } p_{n}\left\{-\frac{1}{n} \log p_{n}(\omega)<a\right\} \leq \epsilon\right\},
\end{array}
$$

for $0 \leq \epsilon<1$, where the $\omega$ is an element of the probability space $\Omega_{n}$.

For example, when the probability $p_{n}$ is the $n$-th independent and identical distribution (i.i.d.) $P^{n}$ of $P$, the law of large numbers guarantees that these quantities coincide with entropy $H(P) \stackrel{\text { def }}{=}-\sum_{\omega} P(\omega) \log P(\omega)$. Therefore, for a more detailed description of asymptotic behavior, we introduce the following quantities.

$$
\begin{array}{r}
\underline{H}(\epsilon, a \mid \bar{p}) \stackrel{\text { def }}{=} \inf _{b}\left\{b \mid \varlimsup p_{n}\left\{-\frac{1}{n} \log p_{n}\left(\omega_{n}\right)<a+\frac{b}{\sqrt{n}}\right\} \geq \epsilon\right\} \\
=\sup _{b}\left\{b \mid \varlimsup p_{n}\left\{-\frac{1}{n} \log p_{n}\left(\omega_{n}\right)<a+\frac{b}{\sqrt{n}}\right\}<\epsilon\right\}, \\
\bar{H}(\epsilon, a \mid \bar{p}) \stackrel{\text { def }}{=} \inf _{b}\left\{b \mid \varliminf p_{n}\left\{-\frac{1}{n} \log p_{n}\left(\omega_{n}\right)<a+\frac{b}{\sqrt{n}}\right\} \geq \epsilon\right\} \\
=\sup _{b}\left\{b \mid \underline{\lim } p_{n}\left\{-\frac{1}{n} \log p_{n}\left(\omega_{n}\right)<a+\frac{b}{\sqrt{n}}\right\}<\epsilon\right\},
\end{array}
$$

for $0<\epsilon \leq 1$. Similarly, $\underline{H}_{+}(\epsilon, a \mid \bar{p})$ and $\bar{H}_{+}(\epsilon, a \mid \bar{p})$ are defined for $0 \leq \epsilon<1$. When the distribution $p_{n}$ is the i.i.d. $P^{n}$ of $P$, the central limit theorem guarantees that $\sqrt{n}\left(-\frac{1}{n} \log P^{n}\left(\omega_{n}\right)-H(P)\right)$ obeys the normal distribution with expectation 0 and variance $V_{P}=$ $\sum_{\omega} P(\omega)(-\log P(\omega)-H(P))^{2}$. Therefore, by using the distribution function $\Phi$ for the standard normal distribution (with expectation 0 and the variance 1 ):

$$
\Phi(x) \stackrel{\text { def }}{=} \int_{-\infty}^{x} \frac{1}{\sqrt{2 \pi}} e^{-x^{2} / 2} d x,
$$


we can express the above quantities as follows:

$$
\begin{aligned}
& \underline{H}(\epsilon, H(P) \mid \bar{P})=\bar{H}(\epsilon, H(P) \mid \bar{P}) \\
= & \underline{H}_{+}(\epsilon, H(P) \mid \bar{P})=\bar{H}_{+}(\epsilon, H(P) \mid \bar{P})=\sqrt{V_{P}} \Phi^{-1}(\epsilon),
\end{aligned}
$$

where $\bar{P}=\left\{P^{n}\right\}$.

In the following, we discuss the relation between the above mentioned quantities, fixed-length source coding, and intrinsic randomness.

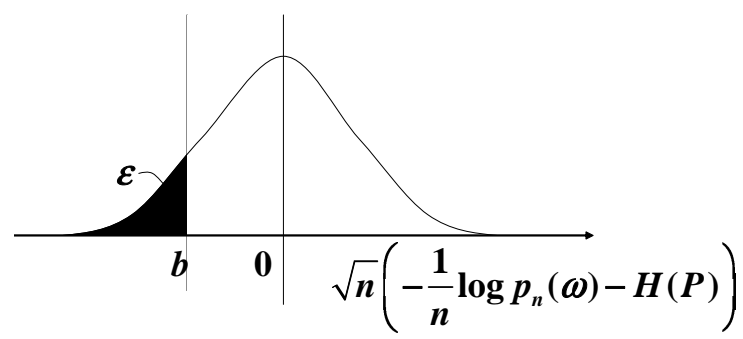

Fig. 1. The limiting distribution of the logarithm of likelihood

\section{FIRST ORDER ASYMPTOTICS}

\section{A. Fixed-length source coding}

In fixed-length source coding, first we fix a set of integers $\mathcal{M}_{n} \stackrel{\text { def }}{=}\left\{1, \ldots, M_{n}\right\}$. The transformation from the output $\omega \in$ $\Omega_{n}$ to an element of the set $\mathcal{M}_{n}$ is described by a map $\phi_{n}$ : $\Omega_{n} \rightarrow \mathcal{M}_{n}$, which is called encoding.

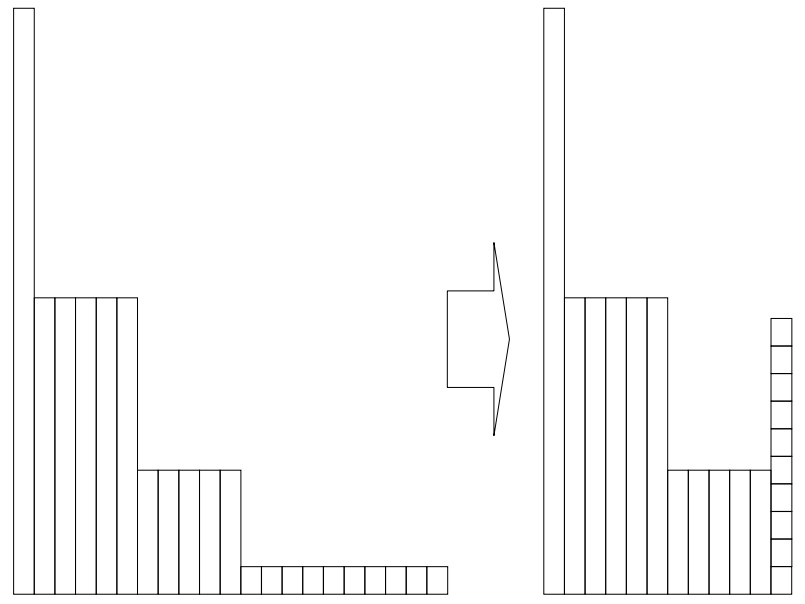

Fig. 2. Encoding operation in source coding

The operation recovering the original output $\omega$ from the element of $\mathcal{M}_{n}$ is described by a map $\psi_{n}: \mathcal{M}_{n} \rightarrow \Omega_{n}$, and is called decoding. We call the triple $\Phi_{n}\left(\mathcal{M}_{n}, \phi_{n}, \psi_{n}\right)$ a code, and evaluate its performance by its size $\left|\Phi_{n}\right| \stackrel{\text { def }}{=}\left|\mathcal{M}_{n}\right|=M_{n}$ and error probability:

$$
\varepsilon_{p_{n}}\left(\Phi_{n}\right) \stackrel{\text { def }}{=} p_{n}\left\{\omega \in \Omega_{n} \mid \psi_{n} \circ \phi_{n}(\omega) \neq \omega\right\} .
$$

When we do not need to express the distribution of information source $p_{n}$, we simplify $\varepsilon_{p_{n}}\left(\Phi_{n}\right)$ to $\varepsilon\left(\Phi_{n}\right)$. In order to discuss the asymptotic bound of compression rate under the constant constraint on the error probability, we focus on the following values:

$$
\begin{array}{r}
R(\epsilon \mid \bar{p}) \stackrel{\text { def }}{=} \inf _{\left\{\Phi_{n}\right\}}\left\{\varlimsup \frac{1}{n} \log \mid \Phi_{n} \| \varlimsup \lim \varepsilon\left(\Phi_{n}\right) \leq \epsilon\right\}, \\
R^{\dagger}(\epsilon \mid \bar{p}) \stackrel{\text { def }}{=} \inf _{\left\{\Phi_{n}\right\}}\left\{\varlimsup \frac{1}{n} \log \mid \Phi_{n} \| \underline{\lim } \varepsilon\left(\Phi_{n}\right) \leq \epsilon\right\}, \\
R^{\ddagger}(\epsilon \mid \bar{p}) \stackrel{\text { def }}{=} \inf _{\left\{\Phi_{n}\right\}}\left\{\underline{\lim } \frac{1}{n} \log \mid \Phi_{n} \| \varlimsup \overline{\lim } \varepsilon\left(\Phi_{n}\right) \leq \epsilon\right\},
\end{array}
$$

for $0 \leq \epsilon<1$, and

$$
\begin{aligned}
& R_{+}(\epsilon \mid \bar{p}) \stackrel{\text { def }}{=} \inf _{\left\{\Phi_{n}\right\}}\left\{\varlimsup \frac{1}{n} \log \left|\Phi_{n}\right| \mid \varlimsup \lim \varepsilon\left(\Phi_{n}\right)<\epsilon\right\}, \\
& R_{+}^{\dagger}(\epsilon \mid \bar{p}) \stackrel{\text { def }}{=} \inf _{\left\{\Phi_{n}\right\}}\left\{\varlimsup \frac{1}{n} \log \left|\Phi_{n}\right| \mid \underline{\lim } \varepsilon\left(\Phi_{n}\right)<\epsilon\right\}, \\
& R_{+}^{\ddagger}(\epsilon \mid \bar{p}) \stackrel{\text { def }}{=} \inf _{\left\{\Phi_{n}\right\}}\left\{\underline{\lim } \frac{1}{n} \log \mid \Phi_{n} \| \varlimsup \lim \varepsilon\left(\Phi_{n}\right)<\epsilon\right\},
\end{aligned}
$$

for $0<\epsilon \leq 1$. Further, as intermediate quantities, we define

$$
\begin{aligned}
& \tilde{R}(\epsilon \mid \bar{p}) \stackrel{\text { def }}{=} \inf _{\left\{\Phi_{n}\right\}}\left\{\varlimsup \frac{1}{n} \log \mid \Phi_{n} \| \varepsilon\left(\Phi_{n}\right) \leq \epsilon, \forall n\right\} \\
&=\inf _{\left\{\Phi_{n}\right\}}\left\{\varlimsup \frac{1}{n} \log \mid \Phi_{n} \| \exists N \varepsilon\left(\Phi_{n}\right) \leq \epsilon, \forall n \geq N\right\}, \\
& \tilde{R}^{\dagger}(\epsilon \mid \bar{p}) \stackrel{\text { def }}{=} \inf _{\left\{\Phi_{n}\right\}}\left\{\frac{\left.1 \lim \frac{1}{n} \log \left|\Phi_{n}\right| \mid \begin{array}{l}
\varepsilon\left(\Phi_{n}\right) \leq \epsilon \\
\text { for infinitely many } n
\end{array}\right\},}{\tilde{R}^{\ddagger}(\epsilon \mid \bar{p}) \stackrel{\text { def }}{=} \inf _{\left\{\Phi_{n}\right\}}\left\{\underline{\lim } \frac{1}{n} \log \mid \Phi_{n} \| \varepsilon\left(\Phi_{n}\right) \leq \epsilon\right\}}\right. \\
&= \inf _{\left\{\Phi_{n}\right\}}\left\{\underline{\lim } \frac{1}{n} \log \mid \Phi_{n} \| \exists N \varepsilon\left(\Phi_{n}\right) \leq \epsilon, \forall n \geq N\right\},
\end{aligned}
$$

for $0<\epsilon<1$. Here, in order to see the relation with existing results, we defined many versions of the optimal coding length. The following relations follow from their definitions:

$$
\begin{aligned}
R(\epsilon \mid \bar{p}) & \leq \tilde{R}(\epsilon \mid \bar{p}) \leq R_{+}(\epsilon \mid \bar{p}), \\
R^{\dagger}(\epsilon \mid \bar{p}) & \leq \tilde{R}^{\dagger}(\epsilon \mid \bar{p}) \leq R_{+}^{\dagger}(\epsilon \mid \bar{p}), \\
R^{\ddagger}(\epsilon \mid \bar{p}) & \leq \tilde{R}^{\ddagger}(\epsilon \mid \bar{p}) \leq R_{+}^{\ddagger}(\epsilon \mid \bar{p}),
\end{aligned}
$$

for $0<\epsilon<1$.

Concerning these quantities, the following theorem holds.

Theorem 1: Han[4, Theorem 1.6.1], Steinberg \& Verdú[8], Chen \& Alajaji [9], Nagaoka \& Hayashi [10] The relations

$$
\begin{aligned}
& R(1-\epsilon \mid \bar{p})=\bar{H}(\epsilon \mid \bar{p}), \\
& R^{\dagger}(1-\epsilon \mid \bar{p})=R^{\ddagger}(1-\epsilon \mid \bar{p})=\underline{H}(\epsilon \mid \bar{p})
\end{aligned}
$$

hold for $0 \leq \epsilon<1$, and the relations

$$
\begin{aligned}
& R_{+}(1-\epsilon \mid \bar{p})=\bar{H}_{+}(\epsilon \mid \bar{p}), \\
& R_{+}^{\dagger}(1-\epsilon \mid \bar{p})=R_{+}^{\ddagger}(1-\epsilon \mid \bar{p})=\underline{H}_{+}(\epsilon \mid \bar{p})
\end{aligned}
$$

hold for $0<\epsilon \leq 1$.

By using the relations (2), (3), and (4), $\tilde{R}(\epsilon \mid \bar{p}), \tilde{R}^{\dagger}(\epsilon \mid \bar{p})$, and $\tilde{R}^{\ddagger}(\epsilon \mid \bar{p})$ are characterized as follows.

Corollary 1:

$$
\begin{aligned}
& \bar{H}(\epsilon \mid \bar{p}) \leq \tilde{R}(1-\epsilon \mid \bar{p}) \leq \bar{H}_{+}(\epsilon \mid \bar{p}), \\
& \underline{H}(\epsilon \mid \bar{p}) \leq \tilde{R}^{\dagger}(1-\epsilon \mid \bar{p}) \leq \underline{H}_{+}(\epsilon \mid \bar{p}), \\
& \underline{H}(\epsilon \mid \bar{p}) \leq \tilde{R}^{\ddagger}(1-\epsilon \mid \bar{p}) \leq \underline{H}_{+}(\epsilon \mid \bar{p}) .
\end{aligned}
$$


Remark 1: Historically, Steinberg \& Verdú[8] derived (9), and Chen \& Alajaji [9] did (10). Han [4] proved the equation $R(1-\epsilon \mid \bar{p})=\bar{H}(\epsilon \mid \bar{p})$. Following these results, Nagaoka \& Hayashi [10] proved $R_{+}^{\dagger}(1-\epsilon \mid \bar{p})=\underline{H}_{+}(\epsilon \mid \bar{p})$. Other relations are proved for the first time in this paper.

The bounds $R_{+}^{\dagger}(1 \mid \bar{p})$ and $R_{+}^{\ddagger}(1 \mid \bar{p})$ are shortest among the above bounds because $R(\epsilon \mid \bar{p}), R^{\dagger}(\epsilon \mid \bar{p}), R^{\ddagger}(\epsilon \mid \bar{p}), \tilde{R}(\epsilon \mid \bar{p})$, $\tilde{R}^{\dagger}(\epsilon \mid \bar{p})$, and $\tilde{R}^{\ddagger}(\epsilon \mid \bar{p})$ are not defined for epsilon $=1$. Hence, the bounds $R_{+}^{\dagger}(1 \mid \bar{p})$ and $R_{+}^{\ddagger}(1 \mid \bar{p})$ are used in the discussion concerning strong converse property.

\section{B. Intrinsic randomness}

Next, we consider the problem of constructing approximately the uniform probability distribution from a biased probability distribution $p_{n}$ on $\Omega_{n}$. We call this problem intrinsic randomness, and discuss it based on (half) the variational distance in this section. Our operation is described by the pair of size $M_{n}$ of the target uniform probability distribution and the map $\phi_{n}$ from $\Omega_{n}$ to $\mathcal{M}_{n} \stackrel{\text { def }}{=}\left\{1, \ldots, M_{n}\right\}$.

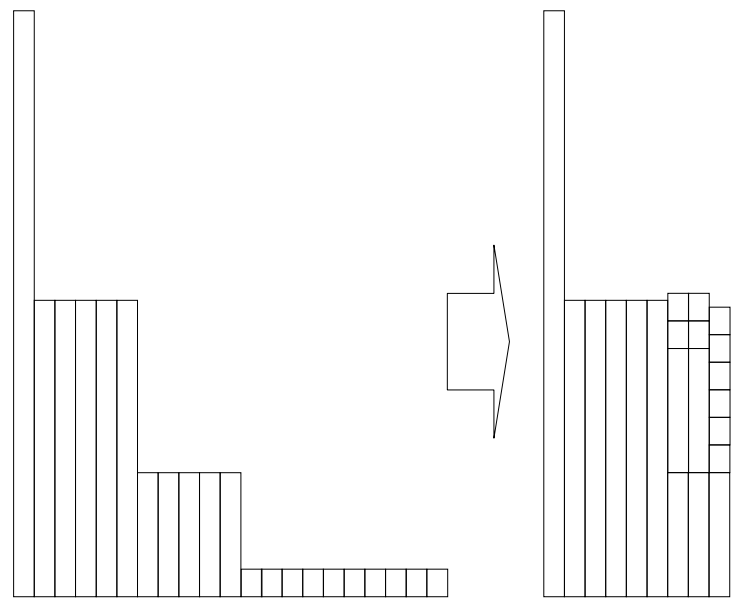

Fig. 3. Typical operation of intrinsic randomness

Performance of $\Psi_{n}=\left(\mathcal{M}_{n}, \phi_{n}\right)$ is characterized by the size $\left|\Psi_{n}\right| \stackrel{\text { def }}{=} M_{n}$ and a half of the variational distance between the target distribution and the constructed distribution:

$$
\varepsilon_{p_{n}}\left(\Psi_{n}\right) \stackrel{\text { def }}{=} d\left(p_{n} \circ \phi_{n}^{-1}, p_{U, \mathcal{M}_{n}}\right),
$$

where $d(p, q) \stackrel{\text { def }}{=} \frac{1}{2} \sum_{\omega}|p(\omega)-q(\omega)|$ and $p_{U, \mathcal{S}}$ is the uniform distribution on $\mathcal{S}$. When we do not need to express the distribution of information source, $p_{n}$, we simplify $\varepsilon_{p_{n}}\left(\Psi_{n}\right)$ to $\varepsilon\left(\Psi_{n}\right)$. Under the condition that this distance is less than $\epsilon$ the optimal size is asymptotically characterized as follows:

$$
\begin{array}{r}
S(\epsilon \mid \bar{p}) \stackrel{\text { def }}{=} \sup _{\left\{\Psi_{n}\right\}}\left\{\underline{\lim } \frac{1}{n} \log \left|\Psi_{n}\right| \mid \lim \varepsilon\left(\Psi_{n}\right)<\epsilon\right\}, \\
S^{\dagger}(\epsilon \mid \bar{p}) \stackrel{\text { def }}{=} \sup _{\left\{\Psi_{n}\right\}}\left\{\underline{\lim } \frac{1}{n} \log \left|\Psi_{n}\right| \mid \underline{\lim } \varepsilon\left(\Psi_{n}\right)<\epsilon\right\}, \\
S^{\ddagger}(\epsilon \mid \bar{p}) \stackrel{\text { def }}{=} \sup _{\left\{\Psi_{n}\right\}}\left\{\varlimsup \frac{1}{n} \log \left|\Psi_{n}\right| \mid \varlimsup \lim \varepsilon\left(\Psi_{n}\right)<\epsilon\right\},
\end{array}
$$

for $0<\epsilon \leq 1$, and

$$
\begin{aligned}
& S_{+}(\epsilon \mid \bar{p}) \stackrel{\text { def }}{=} \sup _{\left\{\Psi_{n}\right\}}\left\{\underline{\lim } \frac{1}{n} \log \left|\Psi_{n}\right| \mid \varlimsup \lim \varepsilon\left(\Psi_{n}\right) \leq \epsilon\right\}, \\
& S_{+}^{\dagger}(\epsilon \mid \bar{p}) \stackrel{\text { def }}{=} \sup _{\left\{\Psi_{n}\right\}}\left\{\underline{\lim } \frac{1}{n} \log \left|\Psi_{n}\right| \mid \underline{\lim } \varepsilon\left(\Psi_{n}\right) \leq \epsilon\right\}, \\
& S_{+}^{\ddagger}(\epsilon \mid \bar{p}) \stackrel{\text { def }}{=} \sup _{\left\{\Psi_{n}\right\}}\left\{\varlimsup \frac{1}{n} \log \left|\Psi_{n}\right| \mid \varlimsup \lim \varepsilon\left(\Psi_{n}\right) \leq \epsilon\right\},
\end{aligned}
$$

for $0 \leq \epsilon<1$. As intermediate quantities,

$$
\begin{aligned}
& \tilde{S}(\epsilon \mid \bar{p}) \stackrel{\text { def }}{=} \sup _{\left\{\Psi_{n}\right\}}\left\{\underline{\lim } \frac{1}{n} \log \mid \Psi_{n} \| \varepsilon\left(\Psi_{n}\right) \leq \epsilon\right\}, \\
& \tilde{S}^{\dagger}(\epsilon \mid \bar{p}) \stackrel{\text { def }}{=} \sup _{\left\{\Psi_{n}\right\}}\left\{\underline{\lim } \frac{1}{n} \log \left|\Psi_{n}\right| \begin{array}{l}
\varepsilon\left(\Psi_{n}\right) \leq \epsilon \\
\text { for infinitely many } n
\end{array}\right\}, \\
& \tilde{S}^{\ddagger}(\epsilon \mid \bar{p}) \stackrel{\text { def }}{=} \sup _{\left\{\Psi_{n}\right\}}\left\{\overline{\lim } \frac{1}{n} \log \left|\Psi_{n}\right| \mid \varepsilon\left(\Psi_{n}\right) \leq \epsilon\right\}
\end{aligned}
$$

are defined for $0<\epsilon<1$. Similarly, we obtain the following trivial relations:

$$
\begin{aligned}
S(\epsilon \mid \bar{p}) & \leq \tilde{S}(\epsilon \mid \bar{p}) \leq S_{+}(\epsilon \mid \bar{p}), \\
S^{\dagger}(\epsilon \mid \bar{p}) & \leq \tilde{S}^{\dagger}(\epsilon \mid \bar{p}) \leq S_{+}^{\dagger}(\epsilon \mid \bar{p}), \\
S^{\ddagger}(\epsilon \mid \bar{p}) & \leq \tilde{S}^{\ddagger}(\epsilon \mid \bar{p}) \leq S_{+}^{\ddagger}(\epsilon \mid \bar{p}),
\end{aligned}
$$

for $0<\epsilon<1$.

These quantities are characterized by the following theorem.

Theorem 2: Han[4, Theorem 2.4.2] The relations

$$
S(\epsilon \mid \bar{p})=\underline{H}(\epsilon \mid \bar{p}), \quad S^{\dagger}(\epsilon \mid \bar{p})=S^{\ddagger}(\epsilon \mid \bar{p})=\bar{H}(\epsilon \mid \bar{p})
$$

hold for $0<\epsilon \leq 1$, and the relations

$$
S_{+}(\epsilon \mid \bar{p})=\underline{H}_{+}(\epsilon \mid \bar{p}), \quad S_{+}^{\dagger}(\epsilon \mid \bar{p})=S_{+}^{\ddagger}(\epsilon \mid \bar{p})=\bar{H}_{+}(\epsilon \mid \bar{p})
$$

hold for $0 \leq \epsilon<1$.

Similarly, the following corollary holds.

Corollary 2: The relations

$$
\begin{aligned}
& \underline{H}(\epsilon \mid \bar{p}) \leq \tilde{S}(\epsilon \mid \bar{p}) \leq \underline{H}_{+}(\epsilon \mid \bar{p}), \\
& \bar{H}(\epsilon \mid \bar{p}) \leq \tilde{S}^{\dagger}(\epsilon \mid \bar{p}) \leq \bar{H}_{+}(\epsilon \mid \bar{p}), \\
& \bar{H}(\epsilon \mid \bar{p}) \leq \tilde{S}^{\ddagger}(\epsilon \mid \bar{p}) \leq \bar{H}_{+}(\epsilon \mid \bar{p})
\end{aligned}
$$

hold for $0<\epsilon<1$.

Remark 2: Han[4] proved only the first equation of (16). Other equations are proved for the first time in this paper.

In the following, in order to treat Folklore for source coding, we focus on the operation $\Psi_{n}=\left(\mathcal{M}_{n}, \phi_{n}\right)$ defined from the code $\Phi_{n}=\left(\mathcal{M}_{n}, \phi_{n}, \psi_{n}\right)$. For fixed real numbers $\epsilon$ and $\epsilon^{\prime}$ satisfying $0 \leq \epsilon, \epsilon^{\prime}<1$, we consider whether there exist codes $\Phi_{n}=\left(\mathcal{M}_{n}, \phi_{n}, \psi_{n}\right)$ such that

$$
\varlimsup=\left(\Phi_{n}\right) \leq \epsilon, \quad \varlimsup \lim \varepsilon\left(\Psi_{n}\right) \leq \epsilon^{\prime} .
$$

If there exists a sequence of codes $\left\{\Phi_{n}\right\}$ satisfying the above conditions, the inequalities

$$
\begin{aligned}
& \underline{H}\left(\epsilon^{\prime} \mid \bar{p}\right)=S\left(\epsilon^{\prime} \mid \bar{p}\right) \geq \underline{\lim } \frac{1}{n} \log M_{n} \geq R(\epsilon \mid \bar{p})=\underline{H}(1-\epsilon \mid \bar{p}), \\
& \bar{H}\left(\epsilon^{\prime} \mid \bar{p}\right)=S^{\ddagger}\left(\epsilon^{\prime} \mid \bar{p}\right) \geq \varlimsup \frac{1}{n} \log M_{n} \geq R^{\ddagger}(\epsilon \mid \bar{p})=\bar{H}(1-\epsilon \mid \bar{p})
\end{aligned}
$$


hold. Therefore, we obtain the following necessary condition for the existence of $\left\{\Phi_{n}\right\}$ satisfying (21):

$$
\underline{H}\left(\epsilon^{\prime} \mid \bar{p}\right) \geq \underline{H}(1-\epsilon \mid \bar{p}), \quad \bar{H}\left(\epsilon^{\prime} \mid \bar{p}\right) \geq \bar{H}(1-\epsilon \mid \bar{p}) .
$$

Thus, the necessary condition (22) is satisfied in the case of i.i.d. $P^{n}$ because these quantities coincide with the entropy $H(P)$.

However, the above discussion is not sufficient, because, as is shown based on the second order asymptotics, a stronger necessary condition exists.

\section{SECOND ORDER ASYMPTOTICS}

Next, we proceed to the second order asymptotics, which is very useful for obtaining the stronger necessary condition than (22). Since these values $\underline{H}\left(\epsilon^{\prime} \mid \bar{p}\right), \bar{H}(\epsilon \mid \bar{p})$ are independent of $\epsilon$ in the i.i.d. case, we introduce the following values for treatment of the dependence of $\epsilon$ :

$$
\begin{aligned}
& R(\epsilon, a \mid \bar{p}) \stackrel{\text { def }}{=} \inf _{\left\{\Phi_{n}\right\}}\left\{\varlimsup \frac{1}{\sqrt{n}} \log \frac{\left|\Phi_{n}\right|}{e^{\text {na }} \mid} \mid \overline{\lim } \varepsilon\left(\Phi_{n}\right) \leq \epsilon\right\}, \\
& R^{\dagger}(\epsilon, a \mid \bar{p}) \stackrel{\text { def }}{=} \inf _{\left\{\Phi_{n}\right\}}\left\{\varlimsup \frac{1}{\sqrt{n}} \log \frac{\left|\Phi_{n}\right|}{e^{n a}} \mid \underline{\lim } \varepsilon\left(\Phi_{n}\right) \leq \epsilon\right\}, \\
& R^{\ddagger}(\epsilon, a \mid \bar{p}) \stackrel{\text { def }}{=} \inf _{\left\{\Phi_{n}\right\}}\left\{\underline{\lim } \frac{1}{\sqrt{n}} \log \frac{\left|\Phi_{n}\right|}{e^{n a}} \mid \overline{\lim } \varepsilon\left(\Phi_{n}\right) \leq \epsilon\right\},
\end{aligned}
$$

for $0 \leq \epsilon<1$, and

$$
\begin{array}{r}
S(\epsilon, a \mid \bar{p}) \stackrel{\text { def }}{=} \sup _{\left\{\Psi_{n}\right\}}\left\{\underline{\lim } \frac{1}{\sqrt{n}} \log \frac{\left|\Psi_{n}\right|}{e^{n a} \mid \varlimsup} \mid \varlimsup\left(\Psi_{n}\right) \leq \epsilon\right\}, \\
S^{\dagger}(\epsilon, a \mid \bar{p}) \stackrel{\text { def }}{=} \sup _{\left\{\Psi_{n}\right\}}\left\{\underline{\lim } \frac{1}{\sqrt{n}} \log \frac{\left|\Psi_{n}\right|}{e^{n a} \mid} \mid \underline{\lim } \varepsilon\left(\Psi_{n}\right) \leq \epsilon\right\}, \\
S^{\ddagger}(\epsilon, a \mid \bar{p}) \stackrel{\text { def }}{=} \sup _{\left\{\Psi_{n}\right\}}\left\{\varlimsup \frac{1}{\sqrt{n}} \log \frac{\left|\Psi_{n}\right|}{e^{n a}} \mid \varlimsup \lim \varepsilon\left(\Psi_{n}\right) \leq \epsilon\right\},
\end{array}
$$

for $0<\epsilon \leq 1$. While we can define other quantities $R_{+}(\epsilon, a \mid \bar{p}), \quad R_{+}^{\dagger}(\epsilon, a \mid \bar{p}), \quad R_{+}^{\ddagger}(\epsilon, a \mid \bar{p}), \quad S_{+}(\epsilon, a \mid \bar{p}), \quad S_{+}^{\ddagger}(\epsilon, a \mid \bar{p})$, and $S_{+}^{\ddagger}(\epsilon, a \mid \bar{p})$, we treat only the above values in this section. This is because the later values can be treated in a similar way. The following theorem holds.

\section{Theorem 3:}

$$
\begin{aligned}
S(\epsilon, a \mid \bar{p}) & =R^{\dagger}(1-\epsilon, a \mid \bar{p})=R^{\ddagger}(1-\epsilon, a \mid \bar{p})=\underline{H}(\epsilon, a \mid \bar{p}), \\
S^{\dagger}(\epsilon, a \mid \bar{p}) & =S^{\ddagger}(\epsilon, a \mid \bar{p})=R(1-\epsilon, a \mid \bar{p})=\bar{H}(\epsilon, a \mid \bar{p}) .
\end{aligned}
$$

Especially, in the case of the i.i.d. $P^{n}$, as is characterized in (1), these quantities with $a=H(P)$ depend on $\epsilon$.

\section{RELATION TO FOLKLORE FOR SOURCE CODING}

Next, we apply Theorem 3 to the relation between the code $\Phi_{n}=\left(\mathcal{M}_{n}, \phi_{n}, \psi_{n}\right)$ and the operation $\Psi_{n}=\left(\mathcal{M}_{n}, \phi_{n}\right)$. When

$$
\varlimsup \varepsilon\left(\Phi_{n}\right)=\epsilon, \quad \varlimsup \lim \varepsilon\left(\Psi_{n}\right)=\epsilon^{\prime},
$$

similar to the previous section, we can derive the following inequalities:

$$
\underline{H}\left(\epsilon^{\prime}, a \mid \bar{p}\right) \geq \underline{H}(1-\epsilon, a \mid \bar{p}), \quad \bar{H}\left(\epsilon^{\prime}, a \mid \bar{p}\right) \geq \bar{H}(1-\epsilon, a \mid \bar{p}) .
$$

Thus, if $\bar{H}\left(\epsilon^{\prime}, a \mid \bar{p}\right)$ or $\underline{H}\left(\epsilon^{\prime}, a \mid \bar{p}\right)$ is continuous with respect to $\epsilon^{\prime}$ at least as in the i.i.d. case, the above equation yields $\epsilon^{\prime} \geq 1-\epsilon$. That is, the following trade-off holds between the error probability of compression and the performance of intrinsic randomness:

$$
\varlimsup
$$

Therefore, Folklore for source coding does not hold with respect to variational distance. In other word, generating completely uniform random numbers requires over compression. Generally, the following theorem holds.

Theorem 4: We define the distance from the uniform distribution as follows:

$$
\delta\left(p_{n}\right) \stackrel{\text { def }}{=} \min _{\mathcal{S} \subset \Omega_{n}} d\left(p_{n}, p_{U, \mathcal{S}}\right) .
$$

Then the following inequality holds:

$$
\varepsilon\left(\Phi_{n}\right)+\varepsilon\left(\Psi_{n}\right) \geq \delta\left(p_{n}\right) .
$$

Especially, in the i.i.d. case, the quantity $\delta\left(p_{n}\right)$ goes to 1 . In such a case, the trade-off relation

$$
\underline{\varliminf}\left(\varepsilon\left(\Phi_{n}\right)+\varepsilon\left(\Psi_{n}\right)\right) \geq 1
$$

holds. Furthermore, the above trade-off inequality (27) is rigid as is indicated by the following theorem.

Theorem 5: When the convergence $\lim _{n \rightarrow \infty} p_{n}\left\{-\frac{1}{n} \log p_{n}\left(\omega_{n}\right)<a+\frac{b+\gamma}{\sqrt{n}}\right\}$ is uniform concerning $\gamma$ in an enough small neibourhood of 0 and the relation

$$
\lim _{\gamma \rightarrow 0} \lim _{n \rightarrow \infty} p_{n}\left\{-\frac{1}{n} \log p_{n}\left(\omega_{n}\right)<a+\frac{b+\gamma}{\sqrt{n}}\right\}=\epsilon
$$

holds, there exists a sequence of codes $\Phi_{n}=\left(\mathcal{M}_{n}, \phi_{n}, \psi_{n}\right)$ $\left(\Psi_{n}=\left(\mathcal{M}_{n}, \phi_{n}\right)\right)$ satisfying the following conditions:

$$
\begin{aligned}
& \varlimsup \varepsilon\left(\Phi_{n}\right) \leq 1-\epsilon, \quad \varlimsup \varepsilon\left(\Psi_{n}\right) \leq \epsilon, \\
& \underline{\lim } \frac{1}{\sqrt{n}} \log \frac{\left|\Phi_{n}\right|}{e^{n a}}=b .
\end{aligned}
$$

\section{INTRINSIC RANDOMNESS BASED ON KL-DIVERGENCE CRITERION}

\section{A. First order asymptotics}

Next, we discuss intrinsic randomness based on KLdivergence. Since Han [1] discussed Folklore for source coding based on KL-divergence criterion, we need this type of discussion for comparing our result and Han's result. The first work on intrinsic randomness based on KL-divergence was done by Vembu \& Verdú[11]. They focused on the normalized KLdivergence:

$$
\frac{1}{n} D\left(p_{n} \circ \phi_{n}^{-1} \| p_{U, \mathcal{M}_{n}}\right),
$$

where $D(p \| q)$ is the KL-divergence $\sum_{\omega} p(\omega) \log \frac{p(\omega)}{q(\omega)}$. Han [1] called the sequence of distributions $p_{n} \circ \phi_{n}^{-1}$ 'almost' uniform random if the above value goes to 0 .

Proposition 1: Vembu \& Verdú[11, Theorem 1]

$$
S^{*}(\bar{p}) \stackrel{\text { def }}{=} \sup _{\Psi_{n}}\left\{\underline{\lim } \frac{1}{n} \log \left|\Psi_{n}\right| \mid \lim \frac{1}{n} D\left(p_{n} \circ \phi_{n}^{-1} \| p_{U, \mathcal{M}_{n}}\right)=0\right\}
$$

$=\underline{H}(\bar{p}) \stackrel{\text { def }}{=} \sup _{a}\left\{a \mid \varlimsup p_{n}\left\{-\frac{1}{n} \log p_{n}(\omega)<a\right\}=0\right\}$. 
In a thorough discussion of the above proposition, Han [1] worked out the following proposition concerning Folklore for source coding.

Proposition 2: Han[1, Theorem 31] The following three conditions for the sequence $\bar{p}=\left\{p_{n}\right\}$ are equivalent:

- When a sequence of codes $\Phi_{n}=\left(\mathcal{M}_{n}, \phi_{n}, \psi_{n}\right)$ satisfies $\varepsilon\left(\Phi_{n}\right) \rightarrow 0, \frac{1}{n} \log \left|\mathcal{M}_{n}\right| \rightarrow \bar{H}(\bar{p})$ then the value (30) goes to 0 .

- There exists a sequence of codes $\Phi_{n}=\left(\mathcal{M}_{n}, \phi_{n}, \psi_{n}\right)$ satisfying $\varepsilon\left(\Phi_{n}\right) \rightarrow 0, \frac{1}{n} \log \left|\mathcal{M}_{n}\right| \rightarrow \bar{H}(\bar{p})$ and the value (30) goes to 0.

- The sequence $\bar{p}=\left\{p_{n}\right\}$ satisfies the strong converse property:

$$
\underline{H}(\bar{p})=\bar{H}(\bar{p}) \stackrel{\text { def }}{=} \inf _{a}\left\{a \mid \underline{\lim } p_{n}\left\{-\frac{1}{n} \log p_{n}(\omega)<a\right\}=1\right\} .
$$

In order to discuss Folklore for source coding in KLdivergence criterion, we need to generalize Vembu \& Verdú's theorem as follows.

Theorem 6: Assume that $\bar{H}(\epsilon \mid \bar{p})=\underline{H}(\epsilon \mid \bar{p})$. We define the probability distribution function $F$ by

$$
\int_{0}^{\underline{H}(\epsilon \mid \bar{p})} F(d x)=\epsilon .
$$

Then, the inequality

$$
\underline{\lim } \frac{1}{n} D\left(p_{n} \circ \phi_{n}^{-1} \| p_{U, \mathcal{M}_{n}}\right) \geq \int_{0}^{a}(a-x) F(d x)
$$

holds, where $a=\underline{\lim } \frac{1}{n} \log M_{n}$. Furthermore, when $\underline{H}(1-$ $\epsilon \mid \bar{p})=a$, there exists a sequence of codes $\left\{\Phi_{n}\right\}$ attaining the equality of (33) and satisfying $\lim \varepsilon\left(\Phi_{n}\right)=\epsilon$. Here, we remark that the inequality 33 is equivalent to the inequality:

$$
\underline{\lim } \frac{1}{n} H\left(p_{n} \circ \phi_{n}^{-1}\right) \leq \int_{0}^{a} x F(d x)+a(1-F(a)) .
$$

Note that the following equation follows from the above theorem:

$$
\begin{aligned}
& S^{*}(\delta \mid \bar{p}) \\
\stackrel{\text { def }}{=} & \sup _{\left\{\Psi_{n}\right\}}\left\{\underline{\lim } \frac{1}{n} \log \left|\Psi_{n}\right| \mid \varlimsup \frac{1}{n} D\left(p_{n} \circ \phi_{n}^{-1} \| p_{U, \mathcal{M}_{n}}\right) \leq \delta\right\} \\
= & \sup _{a}\left\{a \mid \int_{0}^{a}(a-x) F(d x) \leq \delta\right\} .
\end{aligned}
$$

Remark 3: The characterization $S^{*}(\delta \mid \bar{p})$ as a function of $\delta$ was treated as an open problem in Han's textbook [4]. In the i.i.d. case of probability distribution $P$, since

$$
\int_{0}^{a}(a-x) F(d x)= \begin{cases}a-H(P) & a \geq H(P) \\ 0 & a<H(P),\end{cases}
$$

we obtain

$$
S^{*}(\delta \mid \bar{P})=H(P)+\delta
$$

Next, we focus on the opposite criterion:

$$
D\left(p_{U, \mathcal{M}_{n}} \| p_{n} \circ \phi_{n}^{-1}\right),
$$

and define the following quantities:

$$
\begin{aligned}
& S_{1}^{*}(\delta \mid \bar{p}) \\
\stackrel{\text { def }}{=} & \sup _{\left\{\Psi_{n}\right\}}\left\{\underline{\lim } \frac{1}{n} \log \left|\Psi_{n}\right| \mid \varlimsup \lim D\left(p_{U, \mathcal{M}_{n}} \| p_{n} \circ \phi_{n}^{-1}\right)<\delta\right\}, \\
& S_{2}^{*}(\delta \mid \bar{p}) \\
\stackrel{\text { def }}{=} & \left.\sup _{\left\{\Psi_{n}\right\}}\left\{\underline{\lim } \frac{1}{n} \log \left|\Psi_{n}\right|\right\rceil \varlimsup \frac{1}{n} D\left(p_{U, \mathcal{M}_{n}} \| p_{n} \circ \phi_{n}^{-1}\right)<\delta\right\} .
\end{aligned}
$$

Then, they are characterized as follows:

Theorem 7:

$$
S_{1}^{*}(\delta \mid \bar{p})=\underline{H}\left(1-e^{-\delta} \mid \bar{p}\right)
$$

If the limit

$$
\sigma(a) \stackrel{\text { def }}{=} \lim \frac{-1}{n} \log p_{n}\left\{\frac{-1}{n} \log p_{n}(\omega) \geq a\right\}
$$

converges, the relation

$$
S_{2}^{*}(\delta \mid \bar{p})=\sup _{a}\{a-\sigma(a) \mid \sigma(a)<\delta\}
$$

holds for $\forall \delta>0$.

Remark 4: Indeed, Han [4] proved a similar relation concerning the fixed-length source coding with the constraint for error exponent:

$$
\begin{aligned}
& \inf _{\left\{\Phi_{n}\right\}}\left\{\varlimsup \frac{1}{n} \log \left|\Phi_{n}\right| \mid \underline{\lim } \frac{-1}{n} \log \varepsilon\left(\Phi_{n}\right) \geq r\right\} \\
= & \sup _{a}\{a-\underline{\sigma}(a) \mid \underline{\sigma}(a)<r\},
\end{aligned}
$$

where

$$
\underline{\sigma}(a) \stackrel{\text { def }}{=} \underline{\lim } \frac{-1}{n} \log p_{n}\left\{\frac{-1}{n} \log p_{n}(\omega) \geq a\right\} .
$$

Moreover, Nagaoka and Hayashi [10] proved that equation (37) holds when we define $\underline{\sigma}(a)$ by

$$
\underline{\sigma}(a) \stackrel{\text { def }}{=} \underline{\lim } \frac{-1}{n} \log p_{n}\left\{\frac{-1}{n} \log p_{n}(\omega)>a\right\} .
$$

Hence, when the limit $\sigma(a)$ exists, equation (36) holds with replacing $\sigma(a)$ by 38 .

Further, Hayashi [12] showed that when the limit $\sigma(a)$ exists, $\sup _{a}\{a-\sigma(a) \mid \sigma(a) \leq r\}$ is equal to the bound of generation rate of maximally entangled state with the exponential constraint for success probability with the correspondence of each probability to the square of the Schmidt coefficient.

In the i.i.d. case of $P$, these quantities are calculated as

$$
\begin{aligned}
& S_{1}^{*}(\delta \mid \bar{P})=H(P), \\
& S_{2}^{*}(\delta \mid \bar{P})=\min _{0<s \leq 1} \frac{s \delta+\psi(s)}{1-s}, \quad \psi(s) \stackrel{\text { def }}{=} \log \sum_{\omega} P(\omega)^{s},
\end{aligned}
$$

where we use the known value of the left hand side of (37), in the calculation (39).

Remark 5: As is discussed in Theorem 3 of Hayashi [12], when the limit $\bar{\psi}(s):=\lim _{n} \frac{1}{n} \log \sum_{\omega} p_{n}(\omega)^{s}$ and its first and second derivatives $\bar{\psi}^{\prime}(s)$ and $\bar{\psi}^{\prime \prime}(s)$ exist for $s \in(0,1)$, the relation

$$
S_{2}^{*}(\delta \mid \bar{P})=\min _{0<s \leq 1} \frac{s \delta+\bar{\psi}(s)}{1-s}
$$


holds.

From the above discussion, we find that changing the order of input distributions of KL-divergence causes a completely different asymptotic behavior.

\section{B. Second order asymptotics}

Similar to the variational distance criterion, in order to more deeply discuss Folklore for source coding, we need to treat the second order asymptotics. For this purpose, we focus on the following values:

$$
\begin{aligned}
& S^{*}(\delta, a \mid \bar{p}) \\
\stackrel{\text { def }}{=} & \sup _{\left\{\Psi_{n}\right\}}\left\{\underline{\lim } \frac{1}{\sqrt{n}} \log \frac{\left|\Psi_{n}\right|}{e^{n a}} \mid \varlimsup \frac{1}{\sqrt{n}} D\left(p_{n} \circ \phi_{n}^{-1} \| p_{U, \mathcal{M}_{n}}\right) \leq \delta\right\}, \\
& S_{1}^{*}(\delta, a \mid \bar{p}) \\
\stackrel{\text { def }}{=} & \sup _{\left\{\Psi_{n}\right\}}\left\{\underline{\lim } \frac{1}{\sqrt{n}} \log \frac{\left|\Psi_{n}\right|}{e^{n a}} \mid \varlimsup \lim D\left(p_{U, \mathcal{M}_{n}} \| p_{n} \circ \phi_{n}^{-1}\right)<\delta\right\} .
\end{aligned}
$$

Concerning the first value, the following theorem holds.

Theorem 8: Assume that the condition (32) and the equation $\bar{H}(\epsilon, \underline{H}(\bar{p}) \mid \bar{p})=\underline{H}(\epsilon, \underline{H}(\bar{p}) \mid \bar{p})$ hold. Define the probability distribution function $F$ by

$$
\int_{0}^{\underline{H}(\epsilon, \underline{H}(\bar{p}) \mid \bar{p})} F(d x)=\epsilon .
$$

Then, the inequality

$$
\underline{\lim } \frac{1}{\sqrt{n}} D\left(P^{n} \circ \phi_{n}^{-1} \| p_{U, \mathcal{M}_{n}}\right) \geq \int_{-\infty}^{b}(b-x) F(d x)
$$

holds, where $b=\underline{\lim } \frac{1}{\sqrt{n}} \log \frac{M_{n}}{e^{n \underline{H}(\bar{P})}}$. Furthermore, when $\underline{H}(1-\epsilon \underline{H}(\bar{p}) \mid \bar{p})=b$, there exists a sequence of codes $\left\{\Phi_{n}\right\}$ attaining the equality (41) and satisfying $\lim \varepsilon\left(\Phi_{n}\right)=\epsilon$. Finally, we remark that the inequality (41) is equivalent to the inequality:

$\underline{\lim } \frac{1}{\sqrt{n}}\left(H\left(p_{n} \circ \phi_{n}^{-1}\right)-n \underline{H}(\bar{p})\right) \leq \int_{0}^{b} x F(d x)+b(1-F(b))$.

Therefore, we obtain

$$
S^{*}(\delta, \underline{H}(P) \mid \bar{p})=\sup _{b}\left\{b \mid \int_{-\infty}^{b}(b-x) F(d x) \leq \delta\right\} .
$$

Concerning the opposite criterion, the following theorem holds.

Theorem 9:

$$
S_{1}^{*}(\delta, a \mid \bar{p})=\underline{H}\left(1-e^{-\delta}, a \mid \bar{p}\right) .
$$

In the i.i.d. case of $P$, these quantities are simplified to

$$
\begin{aligned}
& S^{*}(\delta, H(P) \mid \bar{P})=\sup _{b}\left\{b \mid \sqrt{V_{P}} \int_{-\infty}^{b} \frac{b-x}{\sqrt{2 \pi}} e^{-x^{2} / 2} d x \leq \delta\right\} \\
& S_{1}^{*}(\delta, H(P) \mid \bar{P})=\sqrt{V_{P}} \Phi^{-1}\left(1-e^{-\delta}\right) .
\end{aligned}
$$

Especially, when we take the limit $\delta \rightarrow 0$, the relations

$$
S^{*}(\delta, H(P) \mid \bar{P}) \rightarrow-\infty, \quad S_{1}^{*}(\delta, H(P) \mid \bar{P}) \rightarrow-\infty
$$

hold. On the other hand, Theorem 3 guarantees that $R^{\ddagger}(\epsilon, a \mid \bar{p})=\underline{H}(1-\epsilon, a \mid \bar{p})$, and $\lim _{\epsilon \rightarrow 0} \underline{H}(1-\epsilon, a \mid \bar{P})=+\infty$.
Thus, if a sequence of codes $\Phi_{n}=\left(\mathcal{M}_{n}, \phi_{n}, \psi_{n}\right)$ satisfies that $\varepsilon\left(\Phi_{n}\right) \rightarrow 0$, it does not satisfy

$$
\frac{1}{\sqrt{n}} D\left(p_{n} \circ \phi_{n}^{-1} \| p_{U, \mathcal{M}_{n}}\right) \rightarrow 0
$$

nor

$$
D\left(p_{U, \mathcal{M}_{n}} \| p_{n} \circ \phi_{n}^{-1}\right) \rightarrow 0 .
$$

Therefore, even if we focus on KL-divergence, if we adopt the criterion (44) or (45), Folklore of source coding does not hold.

Furthermore, combining Theorem 3 we obtain the following corollary.

Corollary 3: Assume the same assumption as Theorem 8 If the function $\epsilon \mapsto \bar{H}(\epsilon, \underline{H}(\bar{p}) \mid \bar{p})$ is continuous, then

$$
\begin{aligned}
& \inf _{\left\{\Phi_{n}\right\}}\left\{\overline{\lim } \frac{1}{\sqrt{n}} D\left(p_{n} \circ \phi_{n}^{-1} \| p_{U, \mathcal{M}_{n}}\right) \mid \varlimsup \lim \varepsilon\left(\Phi_{n}\right) \leq \epsilon\right\} \\
\leq & \inf _{\delta}\left\{\delta \mid S^{*}(\delta, \bar{H}(\bar{p}) \mid \bar{p}) \geq R^{\ddagger}(\epsilon, \bar{H}(\bar{p}) \mid \bar{p})\right\} \\
= & \int_{-\infty}^{F^{-1}(1-\epsilon)}\left(F^{-1}(1-\epsilon)-x\right) F(d x), \\
& \inf _{\left\{\Phi_{n}\right\}}\left\{\overline{\lim } D\left(p_{U, \mathcal{M}_{n}} \| p_{n} \circ \phi_{n}^{-1}\right) \mid \overline{\lim } \varepsilon\left(\Phi_{n}\right) \leq \epsilon\right\} \\
\leq & \inf _{\delta}\left\{\delta \mid S_{1}^{*}(\delta, \bar{H}(\bar{p}) \mid \bar{p}) \geq R^{\ddagger}(\epsilon, \bar{H}(\bar{p}) \mid \bar{p})\right\} \\
= & -\log \epsilon .
\end{aligned}
$$

In the i.i.d. case, the r. h. s. of (46) equals

$$
\begin{aligned}
& \sqrt{V_{P}} \int_{-\infty}^{\sqrt{V_{P}} \Phi^{-1}(1-\epsilon)} \frac{\sqrt{V_{P}} \Phi^{-1}(1-\epsilon)-x}{\sqrt{2 \pi}} e^{-x^{2} / 2} d x \\
= & \int_{-\infty}^{\Phi^{-1}(1-\epsilon)} \frac{\Phi^{-1}(1-\epsilon)-x}{\sqrt{2 \pi}} e^{-x^{2} / 2} d x .
\end{aligned}
$$

Finally, we compare the topologies defined by the following limits:

$$
\begin{aligned}
d\left(p_{n} \circ \phi_{n}^{-1}, p_{U, \mathcal{M}_{n}}\right) & \rightarrow 0 \\
\frac{1}{n} D\left(p_{n} \circ \phi_{n}^{-1} \| p_{U, \mathcal{M}_{n}}\right) & \rightarrow 0 \\
\frac{1}{\sqrt{n}} D\left(p_{n} \circ \phi_{n}^{-1} \| p_{U, \mathcal{M}_{n}}\right) & \rightarrow 0 \\
D\left(p_{n} \circ \phi_{n}^{-1} \| p_{U, \mathcal{M}_{n}}\right) & \rightarrow 0 \\
D\left(p_{U, \mathcal{M}_{n}} \| p_{n} \circ \phi_{n}^{-1}\right) & \rightarrow 0 .
\end{aligned}
$$

The relations

$$
\begin{aligned}
& \text { (50) } \Rightarrow \text { 49) } \Rightarrow \text { 48, } \\
& \text { (50) } \Rightarrow \text { 477 } \Rightarrow \text { 48, } \\
& \text { (51) } \Rightarrow \text { 477) }
\end{aligned}
$$

hold. The relation (52) is trivial, the first relation of (53) and the relation (54) is trivial from Pinsker's inequality. For the second one of (53), see Appendix.

That is, 48 gives the weakest topology among the above topologies. Thus, there is no contradiction, even if Folklore for source coding holds in (48), but does not hold in (49), (51), or 477. 


\section{MARKOVIAN CASE}

Now, we proceed to the Markovian case with irreducible transition matrix $Q_{j, i}$, where $i$ indicates the input signal and $j$ does the output signal. When the initial distribution is the stationary distribution $P_{i}$, which is the eigen vector of $Q_{j, i}$ with eigen value 1 , the average $H_{n}(Q)$ of the normalized likelihood can be calculated as

$$
\begin{aligned}
& H_{n}(Q) \\
= & -\mathrm{E}_{i_{1}, \ldots, i_{n}} \frac{1}{n} \log Q_{i_{n}, i_{n-1}} \cdots Q_{i_{2}, i_{1}} P_{i_{1}} \\
= & -\frac{1}{n} \sum_{i_{n-1}, i_{n}} P_{i_{n-1}} Q_{i_{n}, i_{n-1}} \log Q_{i_{n}, i_{n-1}}+\cdots \\
& +\sum_{i_{1}, i_{2}} P_{i_{1}} Q_{i_{2}, i_{1}} \log Q_{i_{2}, i_{1}}+\sum_{i_{1}} P_{i_{1}} \log P_{i_{1}} \\
= & -\frac{1}{n} \sum_{i} P_{i} \log P_{i}-\frac{n-1}{n} \sum_{j, i} P_{i} Q_{j, i} \log Q_{j, i} \\
\rightarrow & H(Q):=-\sum_{j, i} P_{i} Q_{j, i} \log Q_{j, i},
\end{aligned}
$$

where $\mathrm{E}_{i_{1}, \ldots, i_{n}}$ is the expectation concerning the distribution $Q_{i_{n}, i_{n-1}} \cdots Q_{i_{2}, i_{1}} P_{i_{1}}$.

In oder to treat the limit distribution of the normalized likelihood, we calculate the second cumulant as

$$
\begin{aligned}
& \mathrm{E}_{i_{1}, \ldots, i_{n}}\left(\frac{-\log Q_{i_{n}, i_{n-1}} \cdots Q_{i_{2}, i_{1}} P_{i_{1}}-n H_{n}(Q)}{\sqrt{n}}\right)^{2} \\
= & \mathrm{E}_{i_{1}, \ldots, i_{n}}\left(\frac{X\left(i_{n}, i_{n-1}\right)+\cdots+X\left(i_{2}, i_{1}\right)+Y\left(i_{1}\right)}{\sqrt{n}}\right)^{2} \\
= & \mathrm{E}_{i_{1}, \ldots, i_{n}} \frac{1}{n}\left(X\left(i_{n}, i_{n-1}\right)^{2}+\cdots+X\left(i_{2}, i_{1}\right)^{2}\right. \\
& +Y\left(i_{1}\right)^{2}+2 X\left(i_{n}, i_{n-1}\right) X\left(i_{n-1}, i_{n-2}\right)+\cdots \\
& \left.+2 X\left(i_{3}, i_{2}\right) X\left(i_{2}, i_{1}\right)+2 X\left(i_{2}, i_{1}\right) Y\left(i_{1}\right)\right) \\
\rightarrow & V(Q),
\end{aligned}
$$

where $X\left(i_{k+1}, i_{k}\right):=-\log Q_{i_{k+1}, i_{k}}-H(Q), Y\left(i_{1}\right):=P_{i_{1}}-$ $H(P)$, and

$$
\begin{aligned}
& V(Q) \\
:= & \sum_{j, i} Q_{j, i} P_{i}\left(-\log Q_{j, i}-H(Q)\right)^{2} \\
& +2 \sum_{k, j, i} Q_{k, j} Q_{j, i} P_{i}\left(-\log Q_{k, j}-H(Q)\right)\left(-\log Q_{j, i}-H(Q)\right.
\end{aligned}
$$

The limit of the third cumulant is calculated as

$$
\begin{aligned}
& \mathrm{E}_{i_{1}, \ldots, i_{n}}\left(\frac{-\log Q_{i_{n}, i_{n-1}} \cdots Q_{i_{2}, i_{1}} P_{i_{1}}-n H_{n}(Q)}{\sqrt{n}}\right)^{3} \\
= & \mathrm{E}_{i_{1}, \ldots, i_{n}}\left(\frac{X\left(i_{n}, i_{n-1}\right)+\cdots+X\left(i_{2}, i_{1}\right)+Y\left(i_{1}\right)}{\sqrt{n}}\right)^{3} \\
= & \mathrm{E}_{i_{1}, \ldots, i_{n}} \frac{1}{n \sqrt{n}}\left(X\left(i_{n}, i_{n-1}\right)^{3}+\cdots+X\left(i_{2}, i_{1}\right)^{3}+Y\left(i_{1}\right)^{3}\right. \\
& +3\left(X\left(i_{n}, i_{n-1}\right)^{2} X\left(i_{n-1}, i_{n-2}\right)+\cdots\right. \\
& \left.\quad+X\left(i_{3}, i_{2}\right)^{2} X\left(i_{2}, i_{1}\right)+X\left(i_{2}, i_{1}\right)^{2} Y\left(i_{1}\right)\right) \\
& +3\left(X\left(i_{n}, i_{n-1}\right) X\left(i_{n-1}, i_{n-2}\right)^{2}+\cdots\right. \\
& \left.+X\left(i_{3}, i_{2}\right) X\left(i_{2}, i_{1}\right)^{2}+X\left(i_{2}, i_{1}\right) Y\left(i_{1}\right)\right)^{2} \\
& +2\left(X\left(i_{n}, i_{n-1}\right) X\left(i_{n-1}, i_{n-2}\right) X\left(i_{n-2}, i_{n-3}\right)+\cdots\right. \\
& \left.\left.\quad+X\left(i_{4}, i_{3}\right) X\left(i_{3}, i_{2}\right) X\left(i_{2}, i_{1}\right)+X\left(i_{3}, i_{2}\right) X\left(i_{2}, i_{1}\right) Y\left(i_{1}\right)\right)\right) \\
\rightarrow & 0 .
\end{aligned}
$$

Similarly, for $n \geq 3$, the $n$-th cumulant goes to 0 because the numerator is linear for $n$ while the denominator is a higher term for $n$. Thus, the limit distribution of the normalized likelihood is equal to the normal distribution with average $H(Q)$ and the variance $V(Q)$. Hence, concerning the first order asymptotics, we have

$$
\bar{H}(0 \mid \bar{Q})=\underline{H}_{+}(1 \mid \bar{Q})=H(Q),
$$

where $\bar{Q} \stackrel{\text { def }}{=}\left\{Q^{n}\right\}$ and $Q_{i_{n}, \ldots, i_{1}}^{n} \stackrel{\text { def }}{=} Q_{i_{n}, i_{n-1}} \cdots Q_{i_{2}, i_{1}} P_{i_{1}}$. concerning the second order asymptotics, we have

$$
\underline{H}(\epsilon, H(Q) \mid \bar{Q})=\bar{H}(\epsilon, H(Q) \mid \bar{Q})=\sqrt{V(Q)} \Phi^{-1}(\epsilon) .
$$

Next, we consider the case the initial distribution is the different from the stationary distribution $P_{i}$. In this case, the distribution of the $n$-th data exponentially approaches to the stationary distribution $P_{i}$ [13]. Hence, the limit distribution of the normalized likelihood is equal to the normal distribution with average $H(Q)$ and the variance $V(Q)$. Therefore, Folklore for source coding does not hold for the topology [49, (51), or 477 in the Markovian case as in the i.i.d. case.

Further, by using Remark [5] $\underline{S}_{2}^{*}(\delta \mid \bar{P})$ is calculated as follows. In the Markovian case, $\bar{\psi}(s)=\log \sum_{\omega} Q_{j, i}^{s} P_{s ; i}$, where the distribution $P_{s ; i}$ consists of eigen vectors of the matrix $Q_{j, i}^{s}$ (Section 3 of Dembo \& Zeitouni [13]). Hence, we obtain

$$
S_{2}^{*}(\delta \mid \bar{P})=\min _{0<s \leq 1} \frac{s \delta+\bar{\psi}(s)}{1-s} .
$$

\section{UNIVERSAL FIXED-LENGTH SOURCE CODING AND UNIVERSAL INTRINSIC RANDOMNESS}

In this section, we focus only on the independent and identical information source. In this case, as was shown by Csiszár and Körner[14], there exists a fixed-length source code that attains the first order optimal rate and does not depend on the probability distribution of the information source while we proved the existence of a code attaining the optimal bound depending on the distribution. Such a code is called )universal, and is an important topic in information theory. Indeed, information spectrum method can apply any sequence 
of information source, but gives a code depending on this information source. In contrast, universal code assumes on the independent and identical information source, (or Markovian source), but depends only on the coding rate not on the information source. As is stated in the following theorem, there exists a universal fixed-length source code attaining the second order optimal rate.

Theorem 10: Assume that $|\Omega|$ is a finite number $d$, then there exists a fixed-length source code $\Phi_{n}$ on $\Omega^{n}$ such that

$$
\lim \frac{1}{\sqrt{n}} \frac{\left|\Phi_{n}\right|}{e^{n a}}=b
$$

and

$$
\lim \varepsilon_{P^{n}}\left(\Phi_{n}\right)=\left\{\begin{array}{cc}
0 & H(P)<a \\
1-\Phi\left(\frac{b}{\sqrt{V_{P}}}\right) & H(P)=a .
\end{array}\right.
$$

The error probability of the universal fixed-length source code had not been discussed when the rate equaled the entropy of the information source. But, this theorem clarifies asymptotic behavior of the error probability in such a special case by treating the second order asymptotics.

Concerning intrinsic randomness, while Oohama and Sugano [15] proved that there exists an operation universally attaining the first order optimal rate, we can also prove the existence of a universal operation achieving the second order optimal rate.

Theorem 11: Assume that $|\Omega|$ is a finite number $d$, then there exists an operation $\Psi_{n}$ on $\Omega^{n}$ such that

$$
\lim \frac{1}{\sqrt{n}} \frac{\left|\Phi_{n}\right|}{e^{n a}}=b
$$

and

$$
\lim \varepsilon_{P^{n}}\left(\Psi_{n}\right)=\left\{\begin{array}{cc}
0 & H(P)>a \\
\Phi\left(\frac{b}{\sqrt{V_{P}}}\right) & H(P)=a
\end{array}\right.
$$

\section{ProOF OF THEOREMS}

First, we give proofs of Theorems 11 and 2 which are partially known. Following these proofs, we give our proof of Theorem 3 which is the main result of this paper. This is because the former are preliminaries to our proof of Theorem 3. After these proofs, we give proofs of Theorems 49

\section{A. Proof of Theorem 1}

Lemma 1: Han [4, Lemma 1.3.1] For any integer $M_{n}$, there exists a code $\Phi_{n}$ satisfying

$$
1-\varepsilon\left(\Phi_{n}\right) \geq p_{n}\left\{p_{n}(\omega)>\frac{1}{M_{n}}\right\}, \quad\left|\Phi_{n}\right| \leq M_{n} .
$$

Lemma 2: Han [4, Lemma 1.3.2] Any integer $M_{n}^{\prime}$ and any code $\Phi_{n}$ satisfy the following condition:

$$
1-\varepsilon\left(\Phi_{n}\right) \leq p_{n}\left\{p_{n}(\omega)>\frac{1}{M_{n}^{\prime}}\right\}+\frac{\left|\Phi_{n}\right|}{M_{n}^{\prime}} .
$$

By using these lemmas and the following expressions of the quantities $R(1-\epsilon \mid \bar{p}), R^{\dagger}(1-\epsilon \mid \bar{p})$ and $R^{\ddagger}(1-\epsilon \mid \bar{p})$, we will prove Theorem 1

$$
\begin{aligned}
& R(1-\epsilon \mid \bar{p})=\inf _{\left\{\Phi_{n}\right\}}\left\{\varlimsup \frac{1}{n} \log \left|\Phi_{n}\right| \mid \underline{\lim } 1-\varepsilon\left(\Phi_{n}\right) \geq \epsilon\right\}, \\
& \left.R^{\dagger}(1-\epsilon \mid \bar{p})=\inf _{\left\{\Phi_{n}\right\}}\left\{\varlimsup \frac{1}{n} \log \left|\Phi_{n}\right|\right\rceil \varlimsup i m 1-\varepsilon\left(\Phi_{n}\right) \geq \epsilon\right\}, \\
& R^{\ddagger}(1-\epsilon \mid \bar{p})=\inf _{\left\{\Phi_{n}\right\}}\left\{\underline{\lim } \frac{1}{n} \log \left|\Phi_{n}\right| \mid \underline{\lim } 1-\varepsilon\left(\Phi_{n}\right) \geq \epsilon\right\} .
\end{aligned}
$$

Proof of direct part: For any real number $a>\bar{H}(\epsilon \mid \bar{p})$, by applying Lemma 1 to the case of $M_{n}=e^{n a}$, we can show

$$
\underline{\lim } p_{n}\left\{p_{n}(\omega)>\frac{1}{M_{n}}\right\}=\underline{\lim } p_{n}\left\{-\frac{1}{n} \log p_{n}(\omega)<a\right\} \geq \epsilon,
$$

which implies that $a \geq R(1-\epsilon \mid \bar{p})$. Thus, we obtain

$$
\bar{H}(\epsilon \mid \bar{p}) \geq R(1-\epsilon \mid \bar{p}) \text {. }
$$

By replacing the limit $\underline{\lim }$ in 63 by $\varlimsup$ lim, we can show

$$
\underline{H}(\epsilon \mid \bar{p}) \geq R^{\dagger}(1-\epsilon \mid \bar{p}) \text {. }
$$

Finally, by choosing $M_{n}$ satisfying

$$
\begin{aligned}
& \underline{\lim } p_{n}\left\{-\frac{1}{n} \log p_{n}(\omega)<\frac{1}{n} \log M_{n}\right\} \geq \epsilon \\
& \underline{\lim } \frac{1}{n} \log M_{n}=a>\underline{H}(\epsilon \mid \bar{p}),
\end{aligned}
$$

we can prove

$$
\underline{H}(\epsilon \mid \bar{p}) \geq R^{\ddagger}(1-\epsilon \mid \bar{p}) .
$$

The direct part of (7) and (8) can be proved by replacing $\geq \epsilon$ by $>\epsilon$ in the above proof.

Proof of converse part: First, we prove

$$
\bar{H}(\epsilon \mid \bar{p}) \leq R(1-\epsilon \mid \bar{p}) .
$$

Assume that $a \stackrel{\text { def }}{=} \varlimsup \frac{1}{n} \log \left|\Phi_{n}\right|, \underline{\lim } 1-\varepsilon\left(\Phi_{n}\right) \geq \epsilon$. For any real number $\delta>0$, we apply Lemma 2 to the case of $M_{n}^{\prime}=e^{n(a+\delta)}$. Then, we obtain

$$
p_{n}\left\{-\frac{1}{n} \log p_{n}(\omega)<a+\delta\right\} \geq 1-\varepsilon\left(\Phi_{n}\right)-\frac{\left|\Phi_{n}\right|}{e^{n(a+\delta)}} .
$$

Taking the limit lim, we can show

$$
\underline{\lim } p_{n}\left\{-\frac{1}{n} \log p_{n}(\omega)<a+\delta\right\} \geq \epsilon .
$$

From this relation, we obtain $a+\delta \geq \bar{H}(\epsilon \mid \bar{p})$, which implies (64).

Similarly, taking the limit $\varlimsup$ at 65 , we can prove

$$
\underline{H}(\epsilon \mid \bar{p}) \leq R^{\dagger}(1-\epsilon \mid \bar{p}) \text {. }
$$

Finally, we focus on a subsequence $n_{k}$ satisfying $a \stackrel{\text { def }}{=}$ $\underline{\lim } \frac{1}{n} \log \left|\Phi_{n}\right|=\lim _{k} \frac{1}{n_{k}} \log \left|\Phi_{n_{k}}\right|$. By using 65, we obtain

$$
\begin{aligned}
& \underline{\lim } p_{n}\left\{-\frac{1}{n} \log p_{n}(\omega)<a-\delta\right\} \\
\leq & \lim _{k} p_{n_{k}}\left\{-\frac{1}{n_{k}} \log p_{n_{k}}(\omega)<a-\delta\right\} \leq \lim _{k} 1-\varepsilon\left(\Phi_{n_{k}}\right) .
\end{aligned}
$$

Taking account into the above discussions, we can prove

$$
\underline{H}(\epsilon \mid \bar{p}) \leq R^{\ddagger}(1-\epsilon \mid \bar{p}) .
$$

Similarly, the converse part of (7) and (8) can be proved by replacing $\geq \epsilon$ by $>\epsilon$ in the above proof. 


\section{B. Proof of Theorem 2}

Lemma 3: Han [4, Lemma 2.1.1] For any integers $M_{n}^{\prime}$ and $M_{n}$, there exists an operation $\Psi_{n}=\left(\mathcal{M}_{n}, \phi_{n}\right)$ satisfying

$$
\varepsilon\left(\Psi_{n}\right) \leq p_{n}\left\{p_{n}(\omega)>\frac{1}{M_{n}^{\prime}}\right\}+\frac{M_{n}}{M_{n}^{\prime}},\left|\Psi_{n}\right|=M_{n} .
$$

Lemma 4: Han [4, Lemma 2.1.2] Any integer $M_{n}^{\prime}$ and any operation $\Psi_{n}$ satisfy

$$
\varepsilon\left(\Psi_{n}\right) \geq p_{n}\left\{p_{n}(\omega)>\frac{1}{M_{n}^{\prime}}\right\}-\frac{M_{n}^{\prime}}{\left|\Psi_{n}\right|} .
$$

By using these lemmas, we prove Theorem 2

Proof of direct part: For any real numbers $a<\underline{H}(\epsilon \mid \bar{p})$ and $\delta>0$, we apply Lemma 3 to the case of $M_{n}=$ $e^{n(a-\delta)}, M_{n}^{\prime}=e^{n a}$ as follows:

$$
\begin{aligned}
& \varlimsup p_{n}\left\{p_{n}(\omega)>\frac{1}{M_{n}^{\prime}}\right\} \\
= & \varlimsup p_{n}\left\{-\frac{1}{n} \log p_{n}(\omega)<a\right\}<\epsilon .
\end{aligned}
$$

Since $\frac{M_{n}}{M_{n}^{\prime}} \rightarrow 0$, we obtain $\varlimsup \frac{\lim }{\varepsilon}\left(\Psi_{n}\right)<\epsilon$, which implies that $a-\delta \leq S(\epsilon \mid \bar{p})$. Thus, the inequality

$$
\underline{H}(\epsilon \mid \bar{p}) \leq S(\epsilon \mid \bar{p})
$$

holds. Moreover, by replacing the limit in $\underline{67}$ by $\underline{\lim }$, we can prove

$$
\bar{H}(\epsilon \mid \bar{p}) \leq S^{\dagger}(\epsilon \mid \bar{p})
$$

Finally, by choosing $M_{n}^{\prime}$ satisfying

$$
\begin{aligned}
& \varlimsup p_{n}\left\{-\frac{1}{n} \log p_{n}(\omega)<\frac{1}{n} \log M_{n}^{\prime}\right\}<\epsilon \\
& \varlimsup \frac{1}{n} \log M_{n}^{\prime}=a<\bar{H}(\epsilon \mid \bar{p}),
\end{aligned}
$$

we can prove

$$
\bar{H}(\epsilon \mid \bar{p}) \leq S^{\ddagger}(\epsilon \mid \bar{p}) .
$$

The direct part of (17) can be proved by replacing $<\epsilon$ by $\leq \epsilon$ in the above proof.

Proof of converse part: First, we prove

$$
\underline{H}(\epsilon \mid \bar{p}) \geq S(\epsilon \mid \bar{p}) .
$$

Assume that $a \stackrel{\text { def }}{=} \underline{\lim } \frac{1}{n} \log \left|\Psi_{n}\right|, \varlimsup \lim \epsilon\left(\Psi_{n}\right)<\epsilon$. For any real number $\delta>0$, we apply Lemma 4 to the case of $M_{n}^{\prime}=$ $e^{n(a-\delta)}$. Then, we obtain

$$
p_{n}\left\{-\frac{1}{n} \log p_{n}(\omega)<a-\delta\right\} \leq \varepsilon\left(\Psi_{n}\right)+\frac{e^{n(a-\delta)}}{\left|\Psi_{n}\right|} .
$$

Taking the limit $\varlimsup$ lim, we can show that

$$
\varlimsup p_{n}\left\{-\frac{1}{n} \log p_{n}(\omega)<a-\delta\right\}<\epsilon .
$$

Thus, we obtain $a-\delta \leq \underline{H}(\epsilon \mid \bar{p})$, which implies (68).

Similarly, by taking the limit $\underline{\text { lim }}$ at the inequality $[69$, we obtain

$$
\bar{H}(\epsilon \mid \bar{p}) \geq S^{\dagger}(\epsilon \mid \bar{p}) .
$$

Moreover, by focusing on a subsequence $n_{k}$ satisfying $a \stackrel{\text { def }}{=}$ $\varlimsup \frac{1}{n} \log \left|\Psi_{n}\right|=\lim _{k} \frac{1}{n_{k}} \log \left|\Psi_{n_{k}}\right|$, we can show the following relations from (69):

$$
\begin{aligned}
& \underline{\lim } p_{n}\left\{-\frac{1}{n} \log p_{n}(\omega)<a-\delta\right\} \\
\leq & \lim _{k} p_{n_{k}}\left\{-\frac{1}{n_{k}} \log p_{n_{k}}(\omega)<a-\delta\right\} \leq \lim _{k} \varepsilon\left(\Psi_{n_{k}}\right),
\end{aligned}
$$

which implies that

$$
\bar{H}(\epsilon \mid \bar{p}) \geq S^{\ddagger}(\epsilon \mid \bar{p}) .
$$

Similarly, the converse part of (17) can be proved by replacing $<\epsilon$ by $\leq \epsilon$ in the above proof.

\section{Proof of Theorem 3}

For any real number $b>\bar{H}(\epsilon, a \mid \bar{p})$, by applying Lemma 1 to the case of $M_{n}=e^{n a+\sqrt{n} b}$, we can show

$$
\begin{aligned}
& \underline{\lim } p_{n}\left\{p_{n}(\omega)>\frac{1}{M_{n}}\right\} \\
= & \underline{\lim } p_{n}\left\{-\frac{1}{n} \log p_{n}(\omega)<a+\frac{b}{\sqrt{n}}\right\} \geq \epsilon,
\end{aligned}
$$

which implies $b \geq R(1-\epsilon, a \mid \bar{p})$. Thus, we obtain

$$
\bar{H}(\epsilon, a \mid \bar{p}) \geq R(1-\epsilon, a \mid \bar{p}) .
$$

Similarly to Proof of Theorem 11 we can show

$$
\underline{H}(\epsilon, a \mid \bar{p}) \geq R^{\dagger}(1-\epsilon, a \mid \bar{p}), \quad \underline{H}(\epsilon, a \mid \bar{p}) \geq R^{\ddagger}(1-\epsilon, a \mid \bar{p}) .
$$

Next, we prove

$$
\bar{H}(\epsilon, a \mid \bar{p}) \geq R(1-\epsilon, a \mid \bar{p}) .
$$

Assume that $b \stackrel{\text { def }}{=} \varlimsup \frac{1}{n} \log \frac{\left|\Phi_{n}\right|}{e^{n a}}, \underline{\lim } 1-\epsilon\left(\Phi_{n}\right) \geq \epsilon$. For any real number $\delta>0$, we apply Lemma 2 to the case of $M_{n}^{\prime}=$ $e^{n a+\sqrt{n}(b+\delta)}$. Then, we obtain

$p_{n}\left\{-\frac{1}{n} \log p_{n}(\omega)<a+\frac{b+\delta}{\sqrt{n}}\right\} \geq 1-\varepsilon\left(\Phi_{n}\right)-\frac{\left|\Phi_{n}\right|}{e^{n a+\sqrt{n}(b+\delta)}}$.

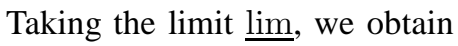

$$
\underline{\lim } p_{n}\left\{-\frac{1}{n} \log p_{n}(\omega)<a+\frac{b+\delta}{\sqrt{n}}\right\} \geq \epsilon,
$$

which implies $b+\delta \geq \bar{H}(\epsilon, a \mid \bar{p})$. Thus, we obtain 70). Therefore, similar to our proof of Theorem 1 we can show

$$
\underline{H}(\epsilon, a \mid \bar{p}) \geq R^{\dagger}(1-\epsilon, a \mid \bar{p}), \quad \underline{H}(\epsilon, a \mid \bar{p}) \geq R^{\ddagger}(1-\epsilon, a \mid \bar{p}) .
$$

Next, we prove

$$
\underline{H}(\epsilon, a \mid \bar{p}) \leq S(\epsilon \mid \bar{p}) .
$$

For any real numbers $b<\underline{H}(\epsilon, a \mid \bar{p})$ and $\delta>0$, we apply Lemma 3 to the case of $M_{n}=e^{n a+\sqrt{n}(b-\delta)}, M_{n}^{\prime}=e^{n a+\sqrt{n} b}$. Since

$$
\begin{aligned}
& \varlimsup p_{n}\left\{p_{n}(\omega)>\frac{1}{M_{n}^{\prime}}\right\} \\
& =\varlimsup p_{n}\left\{-\frac{1}{n} \log p_{n}(\omega)<a+\frac{b}{\sqrt{n}}\right\}<\epsilon
\end{aligned}
$$

and $\frac{M_{n}}{M_{n}^{\prime}} \rightarrow 0$, we obtain $\varlimsup \frac{\lim }{\epsilon}\left(\Psi_{n}\right)<\epsilon$ which implies $a-\delta \leq$ $S(\epsilon \mid \bar{p})$. Thus, we obtain (71). 
Similar to our proof of Theorem 2] we can show

$$
\bar{H}(\epsilon, a \mid \bar{p}) \leq S^{\dagger}(\epsilon, a \mid \bar{p}), \quad \bar{H}(\epsilon, a \mid \bar{p}) \leq S^{\ddagger}(\epsilon, a \mid \bar{p}) .
$$

Finally, we prove

$$
\underline{H}(\epsilon, a \mid \bar{p}) \geq S(\epsilon \mid \bar{p}) .
$$

Assume that $b \stackrel{\text { def }}{=} \underline{\lim } \frac{1}{n} \log \frac{\left|\Psi_{n}\right|}{e^{n a}}$ and $\varlimsup \lim \epsilon\left(\Psi_{n}\right)<\epsilon$. For any real number $\delta>0$, we apply Lemma 4 to the case of $M_{n}^{\prime}=$ $e^{n a+\sqrt{n}(b-\delta)}$. Then, the inequality

$$
p_{n}\left\{-\frac{1}{n} \log p_{n}(\omega)<a+\frac{b-\delta}{\sqrt{n}}\right\} \leq \varepsilon\left(\Psi_{n}\right)+\frac{e^{n a+\sqrt{n}(b-\delta)}}{\left|\Psi_{n}\right|}
$$

holds. Taking the limit $\varlimsup$ lim, we obtain

$$
\varlimsup p_{n}\left\{-\frac{1}{n} \log p_{n}(\omega)<a+\frac{b-\delta}{\sqrt{n}}\right\}<\epsilon,
$$

which implies $b-\delta \leq \underline{H}(\epsilon, a \mid \bar{p})$. Thus, the relation (72) holds.

Similar to our proof of Theorem 2 the inequalities

$$
\bar{H}(\epsilon, a \mid \bar{p}) \geq S^{\dagger}(\epsilon, a \mid \bar{p}), \quad \bar{H}(\epsilon, a \mid \bar{p}) \geq S^{\ddagger}(\epsilon, a \mid \bar{p})
$$

are proved.

\section{Proof of Theorem 4}

We define the subset $\mathcal{M}_{n}^{\prime}$ of $\mathcal{M}_{n}$ as

$$
\mathcal{M}_{n}^{\prime} \stackrel{\text { def }}{=}\left\{i \in \mathcal{M}_{n} \mid \psi_{n}(i) \in \phi_{n}^{-1}(i)\right\}
$$

Since the relation $\phi_{n}^{-1}(i) \cap \phi_{n}^{-1}(j)=\emptyset$ holds for any distinct integers $i, j$, the map $\psi_{n}$ is injective on $\mathcal{M}_{n}^{\prime}$. Thus, $p_{n}$ can be regarded as a probability distribution on $\mathcal{M}_{n}^{\prime} \cup\left(\Omega_{n}\right)$ $\left.\psi_{n}\left(\mathcal{M}_{n}^{\prime}\right)\right) \subset \mathcal{M}_{n} \cup\left(\Omega_{n} \backslash \psi_{n}\left(\mathcal{M}_{n}^{\prime}\right)\right)$. Similarly, $p_{n} \circ \phi_{n}^{-1}$ also can be regarded as a probability distribution on $\mathcal{M}_{n} \subset$ $\mathcal{M}_{n} \cup\left(\Omega_{n} \backslash \psi_{n}\left(\mathcal{M}_{n}^{\prime}\right)\right)$.

Then, the relation

$$
d\left(p_{n}, p_{U, \mathcal{M}_{n}^{\prime}}\right) \leq d\left(p_{n}, p_{U, \mathcal{M}_{n}}\right)
$$

holds. The definition of $\delta\left(p_{n}\right)$ guarantees that

$$
\delta\left(p_{n}\right) \leq d\left(p_{n}, p_{U, \mathcal{M}_{n}^{\prime}}\right)
$$

The axiom of distance yields that

$$
d\left(p_{n}, p_{U, \mathcal{M}_{n}}\right) \leq d\left(p_{n}, p_{n} \circ \phi_{n}^{-1}\right)+d\left(p_{n} \circ \phi_{n}^{-1}, p_{U, \mathcal{M}_{n}}\right) .
$$

Furthermore, the quantity $\varepsilon\left(\Phi_{n}\right)$ has another expression: $\varepsilon\left(\Phi_{n}\right)=p_{n}\left(\Omega_{n} \backslash \psi_{n}\left(\mathcal{M}_{n}^{\prime}\right)\right)$.

Since the set $\left(\Omega_{n} \backslash \psi_{n}\left(\mathcal{M}_{n}^{\prime}\right)\right)$ coincides with the set of the element of $\mathcal{M}_{n} \cup\left(\Omega_{n} \backslash \psi_{n}\left(\mathcal{M}_{n}^{\prime}\right)\right)$ such that the probability $p_{n}$ is greater than the probability $p_{n} \circ \phi_{n}^{-1}$, the equation

$$
d\left(p_{n}, p_{n} \circ \phi_{n}^{-1}\right)=p_{n}\left(\Omega_{n} \backslash \psi_{n}\left(\mathcal{M}_{n}^{\prime}\right)\right)=\varepsilon\left(\Phi_{n}\right)
$$

holds.

Combining the above relations, we obtain

$$
\delta\left(p_{n}\right) \leq \varepsilon\left(\Phi_{n}\right)+\varepsilon\left(\Psi_{n}\right) .
$$

\section{E. Proof of Theorem 5}

First, we construct a sequence of codes $\Phi_{n}=\left(\mathcal{M}_{n}, \phi_{n}, \psi_{n}\right)$ satisfying (28) and (29) as follows. We assume that $S_{n}(a, b) \stackrel{\text { def }}{=}\left\{-\frac{1}{n} \log p_{n}(\omega)<a+\frac{b}{\sqrt{n}}\right\}, \tilde{M}_{n} \stackrel{\text { def }}{=}\left|S_{n}(a, b)\right|$ and denote the one-to-one map from $S_{n}(a, b)$ to $\tilde{\mathcal{M}}_{n} \stackrel{\text { def }}{=}$ $\left\{1, \ldots, \tilde{M}_{n}\right\}$ by $\tilde{\phi}_{n}$.

Then, the inequality $\tilde{M}_{n} \leq e^{n a+\sqrt{n} b}$ holds. Next, we define $\epsilon_{n} \stackrel{\text { def }}{=} p_{n}\left(S_{n}(a, b)\right)$ and focus on the probability distribution $\hat{p}_{n}(\omega) \stackrel{\text { def }}{=} \frac{p_{n}(\omega)}{1-\epsilon_{n}}$ on $S_{n}(a, b)^{c}$. Then, we apply Lemma 3 to the case of $M_{n}^{\prime}=\hat{M}_{n}^{\prime} \stackrel{\text { def }}{=}\left(1-\epsilon_{n}\right) e^{n a+\sqrt{n}\left(b+2 \gamma_{n}\right)}, M_{n}=$ $\hat{M}_{n} \stackrel{\text { def }}{=}\left(1-\epsilon_{n}\right) e^{n a+\sqrt{n}\left(b+\gamma_{n}\right)}, \gamma_{n}=1 / n^{1 / 4}$, and denote the transformation satisfying the condition of Lemma 3 by $\hat{\phi}_{n}$, where the range of $\hat{\phi}_{n}$ is $\left\{\tilde{M}_{n}+1, \ldots, \hat{M}_{n}+\tilde{M}_{n}\right\}$. Half of the variational distance between $\hat{p}_{n} \circ \hat{\phi}_{n}^{-1}$ and the uniform distribution is less than

$$
\hat{p}_{n}\left\{-\frac{1}{n} \log p_{n}(\omega)<a+\frac{b+2 \gamma_{n}}{\sqrt{n}}\right\}+e^{-\sqrt{n} \gamma_{n}} .
$$

Next, we define a code $\Phi_{n}=\left(\mathcal{M}_{n}, \phi_{n}, \psi_{n}\right)$ with the size $M_{n}=e^{n a+\sqrt{n}\left(b+\gamma_{n}\right)}$ as follows. The encoding $\phi_{n}$ is defined by $\tilde{\phi}_{n}$ and $\hat{\phi}_{n}$. The decoding $\psi_{n}$ is defined as the inverse map on the subset $\tilde{\mathcal{M}}_{n}$ of $\mathcal{M}_{n}$, and is defined as an arbitrary map on the compliment set $\tilde{\mathcal{M}}_{n}^{c}$. Since

$$
1-\varepsilon\left(\Phi_{n}\right) \geq p_{n}\left(S_{n}(a, b)\right)=\epsilon_{n},
$$

we obtain the first inequality of (28).

Since the variational distance equals the sum of that on the range of $\hat{\phi}_{n}$ and that on the compliment set of the range, $\varepsilon\left(\Psi_{n}\right)$ can be evaluated as follows:

$$
\begin{aligned}
& \varepsilon\left(\Psi_{n}\right) \\
\leq & \left(1-\epsilon_{n}\right)\left(\hat{p}_{n}\left\{-\frac{1}{n} \log p_{n}(\omega)<a+\frac{b+2 \gamma_{n}}{\sqrt{n}}\right\}+e^{-\sqrt{n} \gamma_{n}}\right) \\
& +p_{n}\left(S_{n}(a, b)\right) \\
= & \left(1-\epsilon_{n}\right) e^{-\sqrt{n} \gamma_{n}}+p_{n}\left(S_{n}(a, b)\right) \\
& +p_{n}\left(S_{n}(a, b)^{c} \cap\left\{-\frac{1}{n} \log p_{n}(\omega)<a+\frac{b+2 \gamma_{n}}{\sqrt{n}}\right\}\right) \\
= & \left(1-\epsilon_{n}\right) e^{-\sqrt{n} \gamma_{n}}+p_{n}\left\{-\frac{1}{n} \log p_{n}(\omega)<a+\frac{b+2 \gamma_{n}}{\sqrt{n}}\right\} \\
\rightarrow & \epsilon,
\end{aligned}
$$

where we use the relation $S_{n}(a, b) \subset\left\{-\frac{1}{n} \log p_{n}(\omega)<a+\right.$ $\left.\frac{b+2 \gamma_{n}}{\sqrt{n}}\right\}$. Since the definition of $M_{n}$ guarantees the condition (29), the proof is completed.

\section{F. Proof of Theorem 6}

Proof of inequality (33):

Lemma 5: The following relation holds for any operation $\left(\mathcal{M}_{n}, \phi_{n}\right)$ :

$$
\begin{aligned}
& H\left(p_{n} \circ \phi_{n}^{-1}\right) \\
\leq & H\left(M_{n}, p_{n}\right) \\
& +p_{n}\left\{p_{n}(\omega) \leq \frac{1}{M_{n}}\right\}\left(\log M_{n}-\log p_{n}\left\{p_{n}(\omega) \leq \frac{1}{M_{n}}\right\}\right),
\end{aligned}
$$


where

$$
H\left(M_{n}, p_{n}\right) \stackrel{\text { def }}{=}-\sum_{p_{n}(\omega)>\frac{1}{M_{n}}} p_{n}(\omega) \log p_{n}(\omega) .
$$

Proof: Define the set $\mathcal{M}_{n}^{\prime}$ and the map $\phi_{n}^{\prime}$ from $\Omega_{n}$ to $\mathcal{M}_{n}^{\prime}$ as follows:

$$
\begin{gathered}
\mathcal{M}_{n}^{\prime} \stackrel{\text { def }}{=} \mathcal{M}_{n} \cup\left\{p_{n}(\omega)>\frac{1}{M_{n}}\right\}, \\
\phi_{n}^{\prime}(\omega) \stackrel{\text { def }}{=} \begin{cases}\phi_{n}(\omega) & p_{n}(\omega) \leq \frac{1}{M_{n}} \\
\omega & p_{n}(\omega)>\frac{1}{M_{n}} .\end{cases}
\end{gathered}
$$

Since

$$
\begin{aligned}
& -\sum_{i=1}^{M_{n}} p_{n} \circ{\phi_{n}^{\prime}}^{-1}(i) \log p_{n} \circ{\phi_{n}^{\prime}}^{-1}(i) \\
\leq & p_{n}\left\{p_{n}(\omega) \leq \frac{1}{M_{n}}\right\}\left(\log M_{n}-\log p_{n}\left\{p_{n}(\omega) \leq \frac{1}{M_{n}}\right\}\right),
\end{aligned}
$$

the inequality

$$
\begin{aligned}
& H\left(p_{n} \circ \phi_{n}^{\prime-1}\right) \\
\leq & H\left(M_{n}, p_{n}\right) \\
& +p_{n}\left\{p_{n}(\omega) \leq \frac{1}{M_{n}}\right\}\left(\log M_{n}-\log p_{n}\left\{p_{n}(\omega) \leq \frac{1}{M_{n}}\right\}\right)
\end{aligned}
$$

holds. When the map $\phi_{n}^{\prime \prime}$ from $\mathcal{M}_{n}^{\prime}$ to $\mathcal{M}_{n}$ is defined by

$$
\phi_{n}^{\prime \prime}(\omega) \stackrel{\text { def }}{=} \begin{cases}\omega & \omega \in \mathcal{M}_{n} \\ \phi_{n}(\omega) & \omega \in\left\{p_{n}(\omega)>\frac{1}{M_{n}}\right\}\end{cases}
$$

the relation $\phi_{n}=\phi_{n}^{\prime \prime} \circ \phi_{n}^{\prime}$ holds. Thus, $\phi_{n}^{-1}={\phi_{n}^{\prime}}^{-1} \circ \phi_{n}^{\prime \prime-1}$. Generally, any map $f$ and any distribution $Q$ satisfies

$$
\begin{aligned}
& H\left(Q \circ f^{-1}\right)=-\sum_{y} \sum_{x: y=f(x)} Q(x) \log \left(\sum_{x^{\prime}: y=f\left(x^{\prime}\right)} Q\left(x^{\prime}\right)\right) \\
\leq & -\sum_{y} \sum_{x: y=f(x)} Q(x) \log Q(x)=H(Q) .
\end{aligned}
$$

Hence,

$$
H\left(p_{n} \circ \phi_{n}^{-1}\right)=H\left(p_{n} \circ \phi_{n}^{\prime-1} \circ \phi_{n}^{\prime \prime-1}\right) \leq H\left(p_{n} \circ \phi_{n}^{\prime-1}\right) .
$$

Therefore, the proof is completed.

We define the probability distribution function $F_{n}$ on the real numbers $\mathbb{R}$ as:

$$
F_{n}(x) \stackrel{\text { def }}{=} p_{n}\left\{-\frac{1}{n} \log p_{n}(\omega)<x\right\}
$$

for a probability distribution $p_{n}$. Then, the relation

$$
\frac{1}{n} H\left(M_{n}, p_{n}\right)=\int_{0}^{\frac{1}{n} \log M_{n}} x F_{n}(d x)
$$

holds. Thus, Lemma 5 yields the inequality

$$
\begin{aligned}
& \frac{1}{n} H\left(p_{n} \circ \phi_{n}^{-1}\right) \\
\leq & \int_{0}^{\frac{1}{n} \log M_{n}} x F_{n}(d x) \\
& +\frac{1}{n} p_{n}\left\{p_{n}(\omega) \leq \frac{1}{M_{n}}\right\}\left(\log M_{n}-\log p_{n}\left\{p_{n}(\omega) \leq \frac{1}{M_{n}}\right\}\right) .
\end{aligned}
$$

Taking the limit, we obtain (34).

Proof of the existence part:
Lemma 6: Han[4, Equation (2.2.4)] For any integers $M_{n}$ and $M^{\prime}{ }_{n}$, there exists an operation $\Psi_{n}$ such that

$$
\begin{aligned}
& D\left(p_{n} \circ \psi^{-1} \| p_{U, \mathcal{M}_{n}}\right) \\
\leq & \log M_{n}\left(\frac{M_{n}^{\prime}{ }_{n}}{M_{n}}+\frac{1}{M_{n}^{\prime}{ }_{n}}+p_{n}\left\{p_{n}(\omega)>\frac{1}{M_{n}}\right\}\right),
\end{aligned}
$$

$\left|\Psi_{n}\right|=M_{n}$

Remark 6: Han [4] derived the above inequality in his proof of Proposition 1

In the following, by using Lemma 6 we construct the code $\Phi_{n}=\left(\mathcal{M}_{n}, \phi_{n}, \psi_{n}\right)$ satisfying the equality of 34 and $\underline{\lim } \varepsilon\left(\Phi_{n}\right)=\epsilon$ as follows. Assume that $S_{n}(a) \stackrel{\text { def }}{=}$ $\left\{-\frac{1}{n} \log p_{n}(\omega)<a\right\}, \tilde{M}_{n} \stackrel{\text { def }}{=}\left|S_{n}(a)\right|$ and let $\tilde{\phi}_{n}$ be the one-to-one map from $S_{n}(a)$ to $\tilde{\mathcal{M}}_{n} \stackrel{\text { def }}{=}\left\{1, \ldots, \tilde{M}_{n}\right\}$. Then, we can prove that $\tilde{M}_{n} \leq e^{n a}$. Moreover, we let $\hat{\phi}_{n}$ be a map satisfying the condition of Lemma 6 for the probability distribution $\hat{p}_{n}(\omega) \stackrel{\text { def }}{=} \frac{p_{n}(\omega)}{1-\epsilon_{n}}$ on the set $S_{n}(a)^{c}$ in the case of $M_{n}=\hat{M}_{n} \stackrel{\text { def }}{=}\left(1-\epsilon_{n}\right) e^{n a}$ and $M^{\prime}{ }_{n}=\sqrt{\hat{M}_{n}}$, where $\epsilon_{n} \stackrel{\text { def }}{=}$ $p_{n}\left(S_{n}(a)\right)$ and the domain of $\hat{\phi}_{n}$ is $\left\{\tilde{M}_{n}+1, \ldots, \hat{M}_{n}+\tilde{M}_{n}\right\}$. Thus,

$$
\begin{aligned}
& D\left(p_{n} \circ \hat{\phi}_{n}^{-1} \| p_{U, \hat{\mathcal{M}}_{n}}\right) \\
\leq & \log \left(\left(1-\epsilon_{n}\right) e^{n a}\right)\left(\hat{p}_{n}\left\{-\frac{1}{n} \log p_{n}(\omega)<a\right\}+\frac{2}{\sqrt{\hat{M}_{n}}}\right) .
\end{aligned}
$$

Since any element of $S_{n}(a)^{c}$ does not satisfy the condition $-\frac{1}{n} \log p_{n}(\omega)<a$, the inequality

$$
H\left(p_{n} \circ \hat{\phi}_{n}^{-1}\right) \geq n a+\log \left(1-\epsilon_{n}\right)-\frac{2\left(n a+\log \left(1-\epsilon_{n}\right)\right)}{\sqrt{\hat{M}_{n}}}
$$

holds.

We define the code $\Phi_{n}=\left(\mathcal{M}_{n}, \phi_{n}, \psi_{n}\right)$ with the size $M_{n}=$ $\tilde{M}_{n}+\hat{M}_{n}$ as follows: The encoding $\phi_{n}$ is defined from $\tilde{\phi}_{n}$ and $\hat{\phi}_{n}$. The decoding $\psi_{n}$ on the subset $\tilde{\mathcal{M}}_{n}$ of $\mathcal{M}_{n}$ is the inverse map of $\tilde{\phi}$. Then, we evaluate $H\left(p_{n} \circ \phi_{n}^{-1}\right)$ as

$$
\begin{aligned}
& H\left(p_{n} \circ \phi_{n}^{-1}\right) \\
= & H\left(e^{n a}, p_{n}\right)+\left(1-\epsilon_{n}\right)\left(H\left(p_{n} \circ \hat{\phi}_{n}^{-1}\right)-\log \left(1-\epsilon_{n}\right)\right) \\
\geq & H\left(e^{n a}, p_{n}\right) \\
& +\left(1-\epsilon_{n}\right)\left(n a-\frac{2\left(n a+\log \left(1-\epsilon_{n}\right)\right)}{\sqrt{\hat{M}_{n}}}\right) \\
= & n \int_{0}^{a} x F_{n}(d x)+n a\left(1-F_{n}(a)\right) \\
& -\frac{2\left(1-\epsilon_{n}\right)\left(n a+\log \left(1-\epsilon_{n}\right)\right)}{\sqrt{\hat{M}_{n}}} .
\end{aligned}
$$

Dividing both sides by $n$ and taking the limit, we obtain the opposite inequality of 41, which implies the inequality of 441. Similar to Theorem 5] we can prove that this code satisfies the condition $\lim \varepsilon\left(\Phi_{n}\right)=\epsilon$.

\section{G. Proof of (35) in Theorem 7}

Proof of direct part: For any real numbers $\epsilon>0$ and $a$ satisfying

$$
a<\underline{H}\left(1-e^{-\delta} \mid \bar{p}\right)
$$


we construct a sequence $\Psi_{n}=\left(\mathcal{M}_{n}, \phi_{n}\right)$ such that

$$
\begin{aligned}
\varlimsup i m\left(p_{U, \mathcal{M}_{n}} \| p_{n} \circ \phi_{n}^{-1}\right) & <\delta \\
\underline{\lim } \frac{1}{n} \log \left|\Psi_{n}\right| & =a-\epsilon .
\end{aligned}
$$

We define the probability distribution $\hat{p}_{n}(\omega) \stackrel{\text { def }}{=} \frac{p_{n}(\omega)}{p_{n}\left(S_{n}(a)^{c}\right)}$ on $S_{n}(a)^{c} \stackrel{\text { def }}{=}\left\{\frac{-1}{n} \log p_{n}(\omega) \geq a\right\}\left(S_{n}(a) \stackrel{\text { def }}{=}\left\{\frac{-1}{n} \log p_{n}(\omega)<\right.\right.$ $a\})$. Since $\hat{p}_{n}\left\{\hat{p}_{n}(\omega)>\frac{e^{-n a}}{p_{n}\left(S_{n}(a)^{c}\right)}\right\}=\hat{p}_{n}\left\{p_{n}(\omega)>e^{-n a}\right\}$ and $S_{n}(a)^{c} \cap\left\{p_{n}(\omega)>e^{-n a}\right\}=\emptyset$, there exists a map from $S_{n}(a)^{c}$ to $\left\{1, \ldots, \hat{M}_{n} \stackrel{\text { def }}{=} e^{n(a-\epsilon)} p_{n}\left(S_{n}(a)^{c}\right)\right\}$ such that the minimum probability of the distribution $\hat{p}_{n} \circ \phi_{n}^{-1}$ is greater than

$$
\frac{1}{\hat{M}_{n}}-\frac{e^{-n a}}{\left.p_{n}\left(S_{n}(a)^{c}\right)\right)}=\frac{1}{\hat{M}_{n}}\left(1-e^{-n \epsilon}\right) .
$$

Hence, we obtain

$$
\begin{aligned}
D\left(p_{U, \hat{\mathcal{M}}_{n}} \| \hat{p}_{n} \circ \hat{\phi}_{n}^{-1}\right) & \leq-\log \frac{1}{\hat{M}_{n}}\left(1-e^{-n \epsilon}\right)+\log \frac{1}{\hat{M}_{n}} \\
& =-\log \left(1-e^{-n \epsilon}\right) \rightarrow 0 .
\end{aligned}
$$

Next, we define a map $\phi_{n}$ from $\Omega_{n}$ to $\mathcal{M}_{n}=$ $\left\{1, \ldots, \hat{M}_{n}, \hat{M}_{n}+1\right\}$ by $\left.\phi_{n}\right|_{S_{n}^{c}(a)}=\hat{\phi}_{n}$ and $\phi_{n}\left(S_{n}(a)\right)=$ $\hat{M}_{n}+1$. Then,

$$
\begin{aligned}
& D\left(p_{U, \mathcal{M}_{n}} \| p_{n} \circ \phi_{n}^{-1}\right) \\
& =-\frac{1}{\hat{M}_{n}+1} \log \left(\hat{M}_{n}+1\right)+\frac{\hat{M}_{n}}{\hat{M}_{n}+1}\left(D\left(p_{U, \hat{\mathcal{M}}_{n}} \| \hat{p}_{n} \circ \hat{\phi}_{n}^{-1}\right)\right. \\
& \left.+\log \frac{\hat{M}_{n}}{\hat{M}_{n}+1}-\log p_{n}\left(S_{n}(a)^{c}\right)\right) \text {. }
\end{aligned}
$$

Since

$$
\varlimsup p_{n}\left(S_{n}(a)\right)<1-e^{-\delta},
$$

we have the inequality 80 that guarantees

$$
\begin{aligned}
& \varlimsup \\
& =\varlimsup-\log p_{n}\left(S_{n}(a)^{c}\right)=\varlimsup \lim -\log \left(1-p_{n}\left(S_{n}(a)\right)\right)<\delta .
\end{aligned}
$$

Moreover,

$$
\begin{aligned}
& \lim \frac{1}{n} \log \left|\mathcal{M}_{n}\right|=\lim \frac{1}{n} \log \left(\hat{M}_{n}+1\right) \\
= & \lim \frac{1}{n} \log \frac{e^{n(a-\epsilon)}}{p_{n}\left(S_{n}(a)^{c}\right)}=a-\epsilon .
\end{aligned}
$$

Proof of converse part: Assume that a sequence $\Psi_{n}=$ $\left(\mathcal{M}_{n}, \phi_{n}\right)$ satisfies

$$
\begin{array}{r}
\underline{\lim } \frac{1}{n} \log \left|\Psi_{n}\right|=R \\
\varlimsup \\
\lim \left(p_{U, \mathcal{M}_{n}} \| p_{n} \circ \phi_{n}^{-1}\right)<\delta .
\end{array}
$$

For any $\epsilon^{\prime}>0$, we define

$$
\begin{aligned}
M_{n}^{\prime} & \stackrel{\text { def }}{=}\left|\left\{\frac{-1}{n} \log p_{n} \circ \phi_{n}^{-1}(i)<R-\epsilon^{\prime}\right\}\right| \\
\epsilon_{n} & \stackrel{\text { def }}{=} p_{n} \circ \phi_{n}^{-1}\left\{\frac{-1}{n} \log p_{n} \circ \phi_{n}^{-1}(i)<R-\epsilon^{\prime}\right\} \\
& \geq p_{n}\left\{\frac{-1}{n} \log p_{n}(\omega)<R-\epsilon^{\prime}\right\} .
\end{aligned}
$$

Information processing inequality of KL-divergence guarantees that

$$
\begin{aligned}
& D\left(p_{U, \mathcal{M}_{n}} \| p_{n} \circ \phi_{n}^{-1}\right) \\
\geq & \frac{M_{n}^{\prime}}{\left|\Phi_{n}\right|}\left(\log \frac{M_{n}^{\prime}}{\left|\Phi_{n}\right|}-\log \epsilon_{n}\right) \\
& +\left(1-\frac{M_{n}^{\prime}}{\left|\Phi_{n}\right|}\right)\left(\log \left(1-\frac{M_{n}^{\prime}}{\left|\Phi_{n}\right|}\right)-\log \left(1-\epsilon_{n}\right)\right) .
\end{aligned}
$$

Since $M_{n}^{\prime} \leq e^{n(R-\epsilon)}$ and 84 ,

$$
\frac{M_{n}^{\prime}}{\left|\Phi_{n}\right|} \rightarrow 0, \quad \frac{M_{n}^{\prime}}{\left|\Phi_{n}\right|} \log \frac{M_{n}^{\prime}}{\left|\Phi_{n}\right|} \rightarrow 0 .
$$

Therefore, taking the limit $\varlimsup$ im, we have

$$
\begin{aligned}
& \delta>\varlimsup \\
&=-\log \left(1-\varlimsup\left(p_{U, \mathcal{M}_{n}} \| p_{n} \circ \phi_{n}^{-1}\right) \geq \varlimsup\right. \\
&\left.\epsilon_{n}\right),
\end{aligned}
$$

which implies

$$
\varlimsup
$$

Thus, inequality 85 yields

$$
\varlimsup p_{n}\left\{\frac{-1}{n} \log p_{n}(\omega)<R-\epsilon^{\prime}\right\}<1-e^{-\delta} .
$$

Therefore,

$$
R-\epsilon^{\prime} \leq \underline{H}\left(1-e^{-\delta} \mid \bar{p}\right) .
$$

Since $\epsilon^{\prime}$ is arbitrary, we obtain

$$
S_{1}^{*}(\delta \mid \bar{p}) \leq \underline{H}\left(1-e^{-\delta} \mid \bar{p}\right)
$$

\section{H. Proof of 36 in Theorem 7} (36).

Lemma 7: When three sequences of positive numbers $a_{n}$, $b_{n}$, and $c_{n}$ satisfy

$$
a_{n} \leq b_{n}+c_{n}
$$

then

$$
\begin{aligned}
& \quad \varlimsup \frac{1}{n} \log a_{n} \leq \max \left\{\varlimsup \frac{1}{n} \log b_{n}, \varlimsup \frac{1}{n} \log c_{n}\right\} . \\
& \text { Lemma } 8: \\
& \sup _{a}\{a-\sigma(a) \mid \sigma(a)<\delta\} \geq \sup _{a}\{\bar{\xi}(a) \mid a-\bar{\xi}(a)<\delta\},
\end{aligned}
$$

where $\bar{\xi}(a)$ is defined as:

$$
\bar{\xi}(a) \stackrel{\text { def }}{=} \varlimsup \frac{1}{n} \log \left|\left\{\frac{-1}{n} \log p_{n}(\omega)<a\right\}\right| .
$$

Proof of direct part: $\quad$ We will prove

$$
S_{2}^{*}(\delta \mid \bar{p}) \geq \sup _{a}\{a-\sigma(a) \mid \sigma(a)<\delta\} .
$$

That is, for any real numbers $\epsilon>0$ and $a$ satisfying $\sigma(a)<\delta$, we construct a sequence $\Psi_{n}=\left(\mathcal{M}_{n}, \phi_{n}\right)$ such that

$$
\begin{aligned}
\varlimsup \frac{1}{n} D\left(p_{U, \mathcal{M}_{n}} \| p_{n} \circ \phi_{n}^{-1}\right) & <\delta \\
\underline{\lim } \frac{1}{n} \log \left|\Psi_{n}\right| & =a-\sigma(a)-\epsilon .
\end{aligned}
$$


Similar to the proof of 35, we define $\hat{p}_{n}(\omega), S_{n}(a)^{c}, S_{n}(a)$ and $\phi_{n}$.

Using 81 and 82, we have

$$
\begin{aligned}
& \lim \frac{1}{n} D\left(p_{U, \mathcal{M}_{n}} \| p_{n} \circ \phi_{n}^{-1}\right) \\
= & \lim \frac{-1}{n} \log p_{n}\left(S_{n}(a)^{c}\right)=\sigma(a)<\delta .
\end{aligned}
$$

Moreover,

$$
\begin{aligned}
& \lim \frac{1}{n} \log \left|\mathcal{M}_{n}\right|=\lim \frac{1}{n} \log \left(\hat{M}_{n}+1\right) \\
= & \lim \frac{1}{n} \log \frac{e^{n(a-\epsilon)}}{p_{n}\left(S_{n}(a)^{c}\right)}=a-\epsilon-\sigma(a) .
\end{aligned}
$$

Proof of converse part: We will prove

$$
S_{2}^{*}(\delta \mid \bar{p}) \leq \sup _{a}\{a-\sigma(a) \mid \sigma(a)<\delta\} .
$$

That is, for any sequence $\Psi_{n}=\left(\mathcal{M}_{n}, \phi_{n}\right)$ satisfying $\varlimsup \frac{1}{n} D\left(p_{U, \mathcal{M}_{n}} \| p_{n} \circ \phi_{n}^{-1}\right) \leq \delta$, we will prove that

$$
R \stackrel{\text { def }}{=} \underline{\lim } \frac{1}{n} \log \left|\mathcal{M}_{n}\right| \leq \sup _{a}\{a-\sigma(a) \mid \sigma(a)<\delta\} .
$$

Let $\left\{n_{k}\right\}$ be a subsequence such that $\lim _{k} \frac{1}{n_{k}} \log \left|\mathcal{M}_{n_{k}}\right|=$ $\underline{\lim } \frac{1}{n} \log \left|\mathcal{M}_{n}\right|$. We choose the real number $a_{0}$

$$
a_{0} \stackrel{\text { def }}{=} \inf \left\{a \mid \varliminf_{k} p_{U, \mathcal{M}_{n}}\left\{\frac{-1}{n_{k}} \log p_{n_{k}} \circ \phi_{n_{k}}^{-1}(i)<a\right\}>0\right\} .
$$

For any real number $\epsilon_{0}>0$, the relation $\underline{\lim }_{k} p_{U, \mathcal{M}_{n_{k}}}\left\{\frac{-1}{n_{k}} \log p_{n} \circ \phi_{n_{k}}^{-1}(i)<a_{0}-\epsilon_{0}\right\}=0$ holds. Since

$$
\begin{aligned}
& n\left(a_{0}-\epsilon_{0}\right) p_{U, \mathcal{M}_{n_{k}}}\left\{\frac{-1}{n_{k}} \log p_{n_{k}} \circ \phi_{n_{k}}^{-1}(i) \geq a_{0}-\epsilon_{0}\right\} \\
\leq & -\sum_{i} p_{U, \mathcal{M}_{n_{k}}}(i) \log p_{n_{k}} \circ \phi_{n_{k}}^{-1}(i),
\end{aligned}
$$

we have

$$
\begin{aligned}
& n\left(a_{0}-\epsilon_{0}\right) p_{U, \mathcal{M}_{n_{k}}}\left\{\frac{-1}{n_{k}} \log p_{n_{k}} \circ \phi_{n_{k}}^{-1}(i) \geq a_{0}-\epsilon_{0}\right\} \\
& \quad-\log M_{n_{k}} \\
\leq & -\log M_{n_{k}}-\sum_{i} p_{U, \mathcal{M}_{n_{k}}}(i) \log p_{n_{k}} \circ \phi_{n_{k}}^{-1}(i) \\
= & D\left(p_{U, \mathcal{M}_{n_{k}}} \| p_{n_{k}} \circ \phi_{n_{k}}^{-1}\right) .
\end{aligned}
$$

Thus,

$$
\begin{aligned}
& \left(a_{0}-\epsilon_{0}\right)-R \\
= & \varlimsup_{k}\left(\left(a_{0}-\epsilon_{0}\right) p_{U, \mathcal{M}_{n_{k}}}\left\{\frac{-1}{n_{k}} \log p_{n} \circ \phi_{n_{k}}^{-1}(i) \geq a_{0}-\epsilon_{0}\right\}\right. \\
& \left.\quad-\frac{1}{n} \log M_{n_{k}}\right) \\
\leq & \varlimsup_{k} \frac{1}{n_{k}} D\left(p_{U, \mathcal{M}_{n_{k}}} \| p_{n_{k}} \circ \phi_{n_{k}}^{-1}\right)<\delta .
\end{aligned}
$$

Taking the limit $\epsilon_{0} \rightarrow 0$,

$$
a_{0}-R \leq \varlimsup_{k} \frac{1}{n_{k}} D\left(p_{U, \mathcal{M}_{n_{k}}} \| p_{n_{k}} \circ \phi_{n_{k}}^{-1}\right)<\delta .
$$

Next, we choose a real number $\epsilon$ such that

$$
0<\epsilon<\delta-\left(a_{0}-R\right) .
$$

Then, there exits a real number $\alpha>0$ such that

$$
\underline{\lim } p_{U, \mathcal{M}_{n_{k}}}\left\{\frac{-1}{n} \log p_{n_{k}} \circ \phi_{n_{k}}^{-1}(i)<a_{0}+\epsilon\right\}>\alpha .
$$

Thus,

$$
\left|\left\{\frac{-1}{n_{k}} \log p_{n_{k}} \circ \phi_{n_{k}}^{-1}(i)<a_{0}+\epsilon\right\}\right|>\alpha M_{n_{k}}
$$

for sufficiently large $n_{k}$. Since

$$
\begin{gathered}
p_{n}\left(\left\{\frac{-1}{n_{k}} \log p_{n_{k}} \circ \phi_{n_{k}}^{-1}(i)<a_{0}+\epsilon\right\}\right. \\
\left.\backslash \phi_{n_{k}}\left\{\frac{-1}{n_{k}} \log p_{n_{k}}(\omega)<a_{0}+\epsilon\right\}\right) \\
\leq p_{n}\left\{\frac{-1}{n_{k}} \log p_{n_{k}}(\omega) \geq a_{0}+\epsilon\right\},
\end{gathered}
$$

we can evaluate

$$
\begin{aligned}
& \quad \mid\left\{\frac{-1}{n_{k}} \log p_{n_{k}} \circ \phi_{n_{k}}^{-1}(i)<a_{0}+\epsilon\right\} \\
& \backslash \phi_{n_{k}}\left\{\frac{-1}{n_{k}} \log p_{n_{k}}(\omega)<a_{0}+\epsilon\right\} \mid \\
& \leq \frac{p_{n}\left\{\frac{-1}{n_{k}} \log p_{n_{k}}(\omega) \geq a_{0}+\epsilon\right\}}{e^{-n\left(a_{0}+\epsilon\right)}} .
\end{aligned}
$$

Thus,

$$
\begin{aligned}
& \alpha M_{n_{k}}<\left|\left\{\frac{-1}{n_{k}} \log p_{n_{k}} \circ \phi_{n_{k}}^{-1}(i)<a_{0}+\epsilon\right\}\right| \\
\leq & \frac{p_{n}\left\{\frac{-1}{n_{k}} \log p_{n_{k}}(\omega) \geq a_{0}+\epsilon\right\}}{e^{-n\left(a_{0}+\epsilon\right)}}+\left|\left\{\frac{-1}{n_{k}} \log p_{n_{k}}(\omega)<a_{0}+\epsilon\right\}\right| .
\end{aligned}
$$

Using Lemma 7 we have

$$
\max \left\{\bar{\xi}\left(a_{0}+\epsilon\right),\left(a_{0}+\epsilon\right)-\sigma\left(a_{0}+\epsilon\right)\right\} \geq R .
$$

If $\bar{\xi}\left(a_{0}+\epsilon\right) \geq\left(a_{0}+\epsilon\right)-\sigma\left(a_{0}+\epsilon\right)$, by combining (88) and 89], we can show

$$
(a+\epsilon)-\bar{\xi}\left(a_{0}+\epsilon\right)<\delta .
$$

Therefore, we obtain

$$
R \leq \sup _{a}\{\bar{\xi}(a) \mid a-\bar{\xi}(a)<\delta\} \leq \sup _{a}\{a-\sigma(a) \mid \sigma(a)<\delta\} .
$$

If $\bar{\xi}\left(a_{0}+\epsilon\right)<\left(a_{0}+\epsilon\right)-\sigma\left(a_{0}+\epsilon\right)$, combining 88 and 89], we can show

$$
\sigma\left(a_{0}+\epsilon\right)<\delta .
$$

Therefore, we obtain

$$
R \leq \sup _{a}\{a-\sigma(a) \mid \sigma(a)<\delta\} .
$$

Proof of Lemma 7 r Since

$$
b_{n}+c_{n} \leq \max \left\{2 b_{n}, 2 c_{n}\right\},
$$


we have

$$
\frac{1}{n} \log a_{n} \leq \max \left\{\frac{\log 2}{n}+\frac{1}{n} \log b_{n}, \frac{\log 2}{n}+\frac{1}{n} \log c_{n}\right\} .
$$

Taking the limit $\varlimsup$,im, we obtain

$$
\varlimsup \frac{1}{n} \log a_{n} \leq \max \left\{\varlimsup \frac{1}{n} \log b_{n}, \varlimsup \frac{1}{n} \log c_{n}\right\} .
$$

Proof of Lemma 8 . In this proof, the following lemma plays an important role.

Lemma 9: Hayashi[12, Lemma 13] If two decreasing functions $f$ and $g$ satisfy

$$
-f(a)+a \geq g(b) \text { if } f(a)>f(b),
$$

then

$$
\sup _{a}\{a-g(a) \mid g(a)<\delta\} \geq \sup _{a}\{f(a) \mid a-f(a)<\delta\} .
$$

Remark 7: This lemma is essentially the one obtained by Hayashi[12]. But, this statement is a little different from Hayashi[12]'s.

Proof: We prove Lemma 9 by reduction to absurdity. Assume that there exists a real number $a_{0}$ such that

$$
\begin{aligned}
a_{0}-f\left(a_{0}\right) & <\delta, \\
f\left(a_{0}\right) & >\sup _{a}\{a-g(a) \mid g(a) \leq r\} .
\end{aligned}
$$

We define $a_{1}:=\inf _{a}\left\{a \mid f(a)=f\left(a_{0}\right)\right\}$ and assume that $a_{0}>$ $a_{1}$. For any real number $\epsilon$ : $0<\epsilon<a_{0}-a_{1}$, the inequality $f\left(a_{1}-\epsilon\right)<f\left(a_{1}+\epsilon\right)$ holds. Using (90), we have

$$
\begin{aligned}
& \quad g\left(a_{1}-\epsilon\right) \leq-f\left(a_{1}+\epsilon\right)+a_{1}+\epsilon=-f\left(a_{0}\right)+a_{1}+\epsilon \\
& <\delta+\left(a_{1}-a_{0}\right)+\epsilon<\delta
\end{aligned}
$$

Thus,

$$
\begin{aligned}
& \sup _{a}\{a-g(a) \mid g(a)<\delta\} \geq a_{1}-\epsilon-g\left(a_{1}-\epsilon\right) \\
\geq & a_{1}-\epsilon-\left(a_{1}+\epsilon\right)+f\left(a_{1}+\epsilon\right)=f\left(a_{0}\right)-2 \epsilon .
\end{aligned}
$$

Taking the limit $\epsilon \rightarrow 0$, we obtain $\sup \{a-g(a) \mid g(a)<r\} \geq$ $f\left(a_{0}\right)$, which contradicts 92 .

Next, we treat the case $a_{0}=a_{1}$. The inequality $f\left(a_{0}\right)>$ $f\left(a_{0}-\epsilon\right)$ holds for $\forall \epsilon>0$. Using (90), we have $g\left(a_{0}-\epsilon\right) \leq$ $-f\left(a_{0}\right)+a_{0} \leq \delta$. Thus,

$$
\begin{aligned}
& \sup _{a}\{a-g(a) \mid g(a) \leq r\} \geq a_{0}-\epsilon-g\left(a_{0}-\epsilon\right) \\
\geq & a_{0}-\epsilon-a_{0}+f\left(a_{0}\right)=-\epsilon+f\left(a_{0}\right) .
\end{aligned}
$$

This also contradicts (92).

Since

$$
\left(p_{n}-e^{n a}\right)\left\{p_{n}-e^{n a} \leq 0\right\} \leq\left(p_{n}-e^{n a}\right)\left\{p_{n}-e^{n b} \leq 0\right\} .
$$

By adding $e^{n a}$ to both sides, we have

$$
\begin{array}{r}
p_{n}\left\{p_{n}-e^{n a} \leq 0\right\}+e^{n a}\left|\left\{p_{n}-e^{n a}>0\right\}\right| \\
\leq p_{n}\left\{p_{n}-e^{n b} \leq 0\right\}+e^{n a}\left|\left\{p_{n}-e^{n b}>0\right\}\right|,
\end{array}
$$

which implies

$$
\begin{aligned}
& \left|\left\{p_{n}-e^{n a}>0\right\}\right| \\
\leq & e^{-n a} p_{n}\left\{p_{n}-e^{n b} \leq 0\right\}+\left|\left\{p_{n}-e^{n b}>0\right\}\right| .
\end{aligned}
$$

Thus, Lemma 7 guarantees that

$$
\bar{\xi}(a) \leq \max \{-a-\sigma(b), \bar{\xi}(b)\} .
$$

Using this relation, we obtain

$$
\bar{\xi}(a) \leq-a-\sigma(b) \text { if } \bar{\xi}(b)<\bar{\xi}(a) .
$$

Therefore, by applying Lemma 9 to the case of $f=\bar{\xi}, g=\sigma$, we can show (86).

\section{Proof of Theorem 8}

Proof of inequality (41): We define the probability distribution function $F_{n}$ on the real numbers $\mathbb{R}$ as:

$$
F_{n}(x) \stackrel{\text { def }}{=} p_{n}\left\{-\frac{1}{n} \log p_{n}(\omega)<\bar{H}(\bar{p})+\frac{x}{\sqrt{n}}\right\}
$$

for a probability distribution $p_{n}$. Then, the relation

$$
H\left(M_{n}, p_{n}\right)=\int_{0}^{b_{n}}(\sqrt{n} x+n \bar{H}(\bar{p})) F_{n}(d x)
$$

holds, where $b_{n} \stackrel{\text { def }}{=} \frac{1}{\sqrt{n}}\left(\log M_{n}-n \bar{H}(\bar{p})\right)$. Thus, Lemma 5 yields the inequality

$$
\begin{aligned}
& H\left(p_{n} \circ \phi_{n}^{-1}\right) \\
\leq & \int_{0}^{b_{n}}(\sqrt{n} x+n \bar{H}(\bar{p})) F_{n}(d x) \\
& +p_{n}\left\{p_{n}(\omega) \leq \frac{1}{M_{n}}\right\} \\
& \quad \times\left(\sqrt{n} b_{n}+n \bar{H}(\bar{p})-\log p_{n}\left\{p_{n}(\omega) \leq \frac{1}{M_{n}}\right\}\right) \\
= & \sqrt{n} \int_{0}^{b_{n}} x F_{n}(d x)+n \bar{H}(\bar{p}) \\
& +p_{n}\left\{p_{n}(\omega) \leq \frac{1}{M_{n}}\right\}\left(\sqrt{n} b_{n}-\log p_{n}\left\{p_{n}(\omega) \leq \frac{1}{M_{n}}\right\}\right) .
\end{aligned}
$$

Therefore, the inequality

$$
\begin{aligned}
& \frac{1}{\sqrt{n}}\left(H\left(p_{n} \circ \phi_{n}^{-1}\right)-n \bar{H}(\bar{p})\right) \\
\leq & \int_{0}^{b_{n}} x F_{n}(d x) \\
& +p_{n}\left\{p_{n}(\omega) \leq \frac{1}{M_{n}}\right\}\left(\sqrt{n} b_{n}-\log p_{n}\left\{p_{n}(\omega) \leq \frac{1}{M_{n}}\right\}\right)
\end{aligned}
$$

holds. Taking the limit lim, we obtain (42), which is equivalent with 41 .

Proof of the existence part: In the following, by using Lemma 6 we construct the code $\Phi_{n}=\left(\mathcal{M}_{n}, \phi_{n}, \psi_{n}\right)$ satisfying the equality at 42 and $\underline{\lim } \varepsilon\left(\Phi_{n}\right)=\epsilon$ as follows. Let $\tilde{\phi}_{n}$ be the one-to-one map from

$$
S_{n}(\bar{H}(\bar{p}), b) \stackrel{\text { def }}{=}\left\{-\frac{1}{n} \log p_{n}(\omega)<\bar{H}(\bar{p})+\frac{b}{\sqrt{n}}\right\}
$$

to $\tilde{\mathcal{M}}_{n} \stackrel{\text { def }}{=}\left\{1, \ldots, \tilde{M}_{n}\right\}$, where $\tilde{M}_{n} \stackrel{\text { def }}{=}\left|S_{n}(\bar{H}(\bar{p}), b)\right|$. Then, the inequality $\tilde{M}_{n} \leq e^{n \bar{H}(\bar{p})+b \sqrt{n}}$ holds. Furthermore, we define $\hat{\phi}_{n}$ as a map satisfying the condition of Lemma 6 for the probability distribution $\hat{p}_{n}(\omega) \stackrel{\text { def }}{=} \frac{p_{n}(\omega)}{1-\epsilon_{n}}$ on the set $S_{n}(\bar{H}(\bar{p}), b)^{c}$ in the case of $M_{n}=\hat{M}_{n} \stackrel{\text { def }}{=}\left(1-\epsilon_{n}\right) e^{n \bar{H}(\bar{p})+b \sqrt{n}}$ 
and $M^{\prime}{ }_{n}=\sqrt{\hat{M}_{n}}$, where $\epsilon_{n} \stackrel{\text { def }}{=} p_{n}\left(S_{n}(\bar{H}(\bar{p}), b)\right)$ and the domain of $\hat{\phi}_{n}$ is $\left\{\tilde{M}_{n}+1, \ldots, \hat{M}_{n}+\tilde{M}_{n}\right\}$. Thus,

$$
\begin{aligned}
& D\left(p_{n} \circ \hat{\phi}_{n}^{-1} \| p_{U, \hat{\mathcal{M}}_{n}}\right) \\
\leq & \log \left(\left(1-\epsilon_{n}\right) e^{n \bar{H}(\bar{p})+b \sqrt{n}}\right) . \\
& \left(\hat{p}_{n}\left\{-\frac{1}{n} \log p_{n}(\omega)<\bar{H}(\bar{p})+\frac{b}{\sqrt{n}}\right\}+\frac{2}{\sqrt{\hat{M}_{n}}}\right) .
\end{aligned}
$$

Because no element of $S_{n}(\bar{H}(\bar{p}), b)^{c}$ satisfies the condition $-\frac{1}{n} \log p_{n}(\omega)<\bar{H}(\bar{p})+\frac{b}{\sqrt{n}}$, the inequality

$$
\begin{aligned}
H\left(p_{n} \circ \hat{\phi}_{n}^{-1}\right) \geq & \log \left(1-\epsilon_{n}\right)+n \bar{H}(\bar{p})+\sqrt{n} b \\
& -\left(n \bar{H}(\bar{p})+\sqrt{n} b+\log \left(1-\epsilon_{n}\right)\right) \frac{2}{\sqrt{\hat{M}_{n}}}
\end{aligned}
$$

holds.

We define the code $\Phi_{n}=\left(\mathcal{M}_{n}, \phi_{n}, \psi_{n}\right)$ with the size $M_{n}=$ $\tilde{M}_{n}+\hat{M}_{n}$ similar to the proof of Theorem $\square$ Then,

$$
\begin{aligned}
& H\left(p_{n} \circ \phi_{n}^{-1}\right) \\
= & H\left(e^{n \bar{H}(\bar{p})+b \sqrt{n}}, p_{n}\right)+\left(1-\epsilon_{n}\right)\left(H\left(p_{n} \circ \hat{\phi}_{n}^{-1}\right)-\log \left(1-\epsilon_{n}\right)\right) \\
\geq & H\left(e^{n \bar{H}(\bar{p})+b \sqrt{n}}, p_{n}\right)+\left(1-\epsilon_{n}\right)(n \bar{H}(\bar{p})+\sqrt{n} b \\
& \left.-\left(n \bar{H}(\bar{p})+\sqrt{n} b+\log \left(1-\epsilon_{n}\right)\right) \frac{2}{\sqrt{\hat{M}_{n}}}\right) \\
= & \sqrt{n} \int_{0}^{b} x F_{n}(d x)+n \bar{H}(\bar{p})+\sqrt{n} b\left(1-F_{n}(b)\right) \\
& -\frac{2\left(1-\epsilon_{n}\right)\left(n \bar{H}(\bar{p})+\sqrt{n} b+\log \left(1-\epsilon_{n}\right)\right)}{\sqrt{\hat{M}_{n}}} .
\end{aligned}
$$

By substracting $n \bar{H}(\bar{p})$ from both sides, dividing both by $\sqrt{n}$, and taking the limit, we obtain the opposite inequality of [42, which implies the inequality of [42]. Similar to Theorem [5] we can prove that this code satisfies the condition $\lim \varepsilon\left(\Phi_{n}\right)=\epsilon$.

\section{J. Proof of Theorem 9}

Proof of direct part: For for any real numbers $\epsilon>0$ and $a$ satisfying

$$
b<\underline{H}\left(1-e^{-\delta}, a \mid \bar{p}\right),
$$

we construct a sequence $\Psi_{n}=\left(\mathcal{M}_{n}, \phi_{n}\right)$ such that

$$
\begin{aligned}
& \varlimsup \overline{l i m} D\left(p_{U, \mathcal{M}_{n}} \| p_{n} \circ \phi_{n}^{-1}\right)<\delta \\
& \underline{\lim } \frac{1}{\sqrt{n}} \log \frac{\left|\Psi_{n}\right|}{e^{n a}}=b-\epsilon .
\end{aligned}
$$

We define the probability distribution $\hat{p}_{n}(\omega) \stackrel{\text { def }}{=} \frac{p_{n}(\omega)}{p_{n}\left(S_{n}(a, b)^{c}\right)}$ on $S_{n}(a, b)^{c} \stackrel{\text { def }}{=}\left\{\frac{-1}{n} \log p_{n}(\omega) \geq a+\frac{b}{\sqrt{n}}\right\} \quad\left(S_{n}(a, b) \stackrel{\text { def }}{=}\right.$ $\left.\left\{\frac{-1}{n} \log p_{n}(\omega)<a+\frac{b}{\sqrt{n}}\right\}\right)$. Then, for any $\epsilon>0$, similar to our proof of (35) in Theorem 7 there exists an operation $\hat{\phi}_{n}$ from $S_{n}(a, b)^{c}$ to $\hat{\mathcal{M}}_{n} \stackrel{\text { def }}{=} e^{n a+\sqrt{n}(b-\epsilon)} p_{n}\left(S_{n}(a, b)^{c}\right)$ such that

$$
D\left(p_{U, \hat{\mathcal{M}}_{n}} \| \hat{p}_{n} \circ \hat{\phi}_{n}^{-1}\right) \leq-\log \left(1-e^{-\epsilon \sqrt{n}}\right) \rightarrow 0 .
$$

Next, we define a map $\phi_{n}$ from $\Omega_{n}$ to $\mathcal{M}_{n}=$ $\left\{1, \ldots, \hat{M}_{n}, \hat{M}_{n}+1\right\}$ by $\left.\phi_{n}\right|_{S_{n}^{c}(a, b)}=\hat{\phi}_{n}$ and $\phi_{n}\left(S_{n}(a, b)\right)=$ $\hat{M}_{n}+1$. Then, we obtain

$$
\begin{aligned}
& D\left(p_{U, \mathcal{M}_{n}} \| p_{n} \circ \phi_{n}^{-1}\right)
\end{aligned}
$$

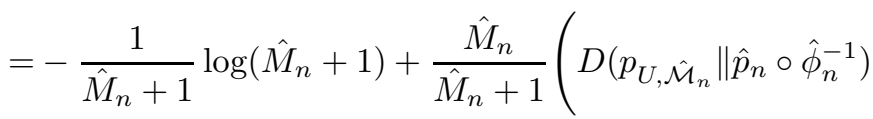

$$
\begin{aligned}
& \left.+\log \frac{\hat{M}_{n}}{\hat{M}_{n}+1}-\log p_{n}\left(S_{n}(a, b)^{c}\right)\right) .
\end{aligned}
$$

Since the inequality (95) guarantees

$$
\varlimsup p_{n}\left(S_{n}(a, b)\right)<1-e^{-\delta},
$$

we have

$\varlimsup \overline{l i m}\left(p_{U, \mathcal{M}_{n}} \| p_{n} \circ \phi_{n}^{-1}\right)$

$=\varlimsup \lim -\log p_{n}\left(S_{n}(a, b)^{c}\right)=\varlimsup \lim -\log \left(1-p_{n}\left(S_{n}(a, b)\right)\right)<\delta$.

Moreover,

$$
\begin{aligned}
& \lim \frac{1}{\sqrt{n}} \log \left|\mathcal{M}_{n}\right|=\lim \frac{1}{\sqrt{n}} \log \left(\hat{M}_{n}+1\right) \\
= & \lim \frac{1}{\sqrt{n}} \log \frac{e^{n(a-\epsilon)}}{p_{n}\left(S_{n}(a, b)^{c}\right)}=b-\epsilon .
\end{aligned}
$$

Proof of converse part: Assume that a sequence $\Psi_{n}=$ $\left(\mathcal{M}_{n}, \phi_{n}\right)$ satisfies

$$
\begin{aligned}
& \underline{\varliminf} \frac{1}{\sqrt{n}} \log \frac{\left|\Psi_{n}\right|}{e^{n a}}=R \\
& \varlimsup \overline{l i m} D\left(p_{U, \mathcal{M}_{n}} \| p_{n} \circ \phi_{n}^{-1}\right)<\delta .
\end{aligned}
$$

For any $\epsilon^{\prime}>0$, we define

$$
\begin{aligned}
& M_{n}^{\prime} \stackrel{\text { def }}{=}\left|\left\{\frac{-1}{n} \log p_{n} \circ \phi_{n}^{-1}(i)<a+\frac{R-\epsilon^{\prime}}{\sqrt{n}}\right\}\right| \\
& \epsilon_{n} \stackrel{\text { def }}{=} p_{n} \circ \phi_{n}^{-1}\left\{\frac{-1}{n} \log p_{n} \circ \phi_{n}^{-1}(i)<a+\frac{R-\epsilon^{\prime}}{\sqrt{n}}\right\} \\
& \quad \geq p_{n}\left\{\frac{-1}{n} \log p_{n}(\omega)<a+\frac{R-\epsilon^{\prime}}{\sqrt{n}}\right\} .
\end{aligned}
$$

Information processing inequality of KL-divergence guarantees that

$$
\begin{aligned}
& D\left(p_{U, \mathcal{M}_{n}} \| p_{n} \circ \phi_{n}^{-1}\right) \\
\geq & \frac{M_{n}^{\prime}}{\left|\Phi_{n}\right|}\left(\log \frac{M_{n}^{\prime}}{\left|\Phi_{n}\right|}-\log \epsilon_{n}\right) \\
& +\left(1-\frac{M_{n}^{\prime}}{\left|\Phi_{n}\right|}\right)\left(\log \left(1-\frac{M_{n}^{\prime}}{\left|\Phi_{n}\right|}\right)-\log \left(1-\epsilon_{n}\right)\right) .
\end{aligned}
$$

Since $M_{n}^{\prime} \leq e^{n a+\sqrt{n}(R-\epsilon)}$ and 97,

$$
\frac{M_{n}^{\prime}}{\left|\Phi_{n}\right|} \rightarrow 0, \quad \frac{M_{n}^{\prime}}{\left|\Phi_{n}\right|} \log \frac{M_{n}^{\prime}}{\left|\Phi_{n}\right|} \rightarrow 0 .
$$

Therefore, taking the limit $\varlimsup$, we have

$$
\begin{aligned}
& \delta>\varlimsup \\
&=-\log \left(1-\varlimsup\left(p_{U, \mathcal{M}_{n}} \| p_{n} \circ \phi_{n}^{-1}\right) \geq \varlimsup\right. \\
&\left.\epsilon_{n}\right),
\end{aligned}
$$


which implies

$$
\varlimsup \overline{\lim } \epsilon_{n}<1-e^{-\delta} .
$$

Thus, the inequality 98 yields

$$
\varlimsup p_{n}\left\{\frac{-1}{n} \log p_{n}(\omega)<R-\epsilon^{\prime}\right\}<1-e^{-\delta} .
$$

Therefore,

$$
R-\epsilon^{\prime} \leq \underline{H}\left(1-e^{-\delta}, a \mid \bar{p}\right) .
$$

Since $\epsilon^{\prime}$ is arbitrary, we obtain

$$
S_{1}^{*}(\delta, a \mid \bar{p}) \leq \underline{H}\left(1-e^{-\delta}, a \mid \bar{p}\right) .
$$

\section{K. Proof of Theorem 10}

This theorem is proved by the type method. Let $\mathcal{T}_{n}$ be the set of $n$-th types, i.e., the set of empirical distributions of $n$ observations. We denote the set of elements $\Omega^{n}$ corresponding to $P$ by $T_{P}^{n} \subset \Omega^{n}$, and define a subset $T_{n}(a, b)$ of the set $\Omega^{n}$ as

$$
T_{n}(a, b) \stackrel{\text { def }}{=} \cup_{P \in \mathcal{T}_{n}:\left|T_{P}^{n}\right| \leq e^{a n+b \sqrt{n}} T_{P}^{n} .}
$$

Using this notation, we define the encoding $\psi_{n}$ from $\Omega^{n}$ to $T_{n}(a, b) \cup\{0\}$ :

$$
\omega \mapsto\left\{\begin{array}{ll}
\omega & \text { if } \omega \in T_{n}(a, b) \\
0 & \text { if } \omega \notin T_{n}(a, b)
\end{array} .\right.
$$

We also define the decoding $\psi_{n}$ such that $\psi_{n}(\omega)=\omega, \forall \omega \in$ $T_{n}(a, b)$. The relation

$$
\varepsilon_{P^{n}}\left(\Phi_{n}\right)=1-P^{n}\left(T_{n}(a, b)\right)
$$

holds. Then, the type counting lemma guarantees that

$$
\left|T_{n}(a, b)\right| \leq(n+1)^{d} e^{a n+b \sqrt{n}},
$$

which implies

$$
\liminf \frac{1}{\sqrt{n}} \log \frac{\left|T_{n}(a, b)\right|}{e^{n a}} \leq b .
$$

On the other hand, the set $\left\{-\log P^{n}(\omega)<n a+b \sqrt{n}\right\}$ can be expressed as

$$
\begin{aligned}
& \left\{-\log P^{n}(\omega)<n a+b \sqrt{n}\right\}=\left\{P^{n}(\omega)>e^{-n a-b \sqrt{n}}\right\} \\
& \bigcup_{P^{\prime} \in \mathcal{T}_{n}: P^{n}(\omega)>e^{-n a-b \sqrt{n}} \text { for } \omega \in T_{P^{\prime}}^{n}} T_{P}^{n} .
\end{aligned}
$$

Hence, when a type $P^{\prime} \in \mathcal{T}_{n}$ satisfies $P^{n}(\omega)>e^{-n a-b \sqrt{n}}$ for $\omega \in T_{P^{\prime}}^{n}$, the inequality $P^{n}\left(T_{P^{\prime}}^{n}\right) \leq 1$ yields

$$
\left|T_{P^{\prime}}^{n}\right| \leq P^{n}(\omega)^{-1} \leq e^{n a+b \sqrt{n}} .
$$

Thus,

$$
\left\{-\log P^{n}(\omega)<n a+b \sqrt{n}\right\} \subset T_{n}(a, b) .
$$

Therefore, if the probability distribution $P$ satisfies $H(P)=a$, then

$$
\begin{aligned}
& \Phi\left(\frac{b}{\sqrt{V_{P}}}\right)=\lim P^{n}\left\{-\log P^{n}(\omega)<n a+b \sqrt{n}\right\} \\
\leq & \lim P^{n}\left(T_{n}(a, b)\right)=1-\lim \varepsilon_{P^{n}}\left(\Phi_{n}\right),
\end{aligned}
$$

i.e.,

$$
\lim \varepsilon_{P^{n}}\left(\Phi_{n}\right) \leq 1-\Phi\left(\frac{b}{\sqrt{V_{P}}}\right) .
$$

Since the r.h.s. of (100) is optimal under the condition 99, the inequality of 100 holds. Conversely, Since the r.h.s. of (99) is optimal under the condition (100), the inequality of (99) holds. Thus, we obtain (59).

In the universal variable-length source code, the order of the second term regarding expected coding length is $\log n$. But, as discussed in the above proof, this term is negligible concerning the second order asymptotics of fixed-length source coding.

Thus, in the variable-length and fixed-length source coding, the central limit theorem plays an important role, while its applications to the respective problems are different.

\section{Proof of Theorem 11}

Using the type method, we define a map $\phi_{n}$ from $\Omega^{n}$ to $\mathcal{M}_{n} \stackrel{\text { def }}{=}\left\{1, \ldots, \frac{1}{n} e^{n a+b \sqrt{n}}\right\}$ as follows. The map $\phi_{n}$ maps any element of $T_{n}(a, b)$ to 1 . On the other hand, the map $\psi$ restricted to a subset $T_{P^{\prime}}^{n} \subset T_{n}(a, b)^{c}$ is defined as the map from $T_{P^{\prime}}^{n}$ to $\mathcal{M}_{n}$ satisfying the conditions Lemma 3 in the case of $M_{n}^{\prime}=\left|T_{P^{\prime}}^{n}\right|$.

Then, the equality of 100 guarantees

$$
\begin{aligned}
& \quad \varepsilon_{P^{n}}\left(\Psi_{n}\right) \\
& \leq \sum_{T_{P^{\prime}}^{n} \subset T_{n}(a, b)^{c}} P^{n}\left(T_{P^{\prime}}^{n}\right) \frac{e^{n a+b \sqrt{n}}}{n\left|T_{P^{\prime}}^{n}\right|}+\sum_{T_{P^{\prime}}^{n} \subset T_{n}(a, b)} P^{n}\left(T_{P^{\prime}}^{n}\right) \\
& \leq P^{n}\left(T_{n}(a, b)^{c}\right) \frac{1}{n}+P^{n}\left(T_{n}(a, b)\right) \\
& \rightarrow\left\{\begin{array}{cc}
0 & H(P)>a \\
\Phi\left(\frac{b}{\sqrt{V_{P}}}\right) & H(P)=a .
\end{array}\right.
\end{aligned}
$$

Therefore, we obtain 61).

\section{CONCluding REMARKS AND Future STUdy}

We proved that Folklore for source coding does not hold for the variational distance criterion (12) nor the KL-divergence criterion (44) nor (45). Of course, since our criteria (12), (44) and (45) are more restrictive than Han's criterion (30), there is no contradiction. But, it is necessary to discuss which criterion is more suitable for treating Folklore for source coding. This is left to future research.

While we focused on the relation between source coding and intrinsic randomness only in the fixed-length case, the compression scheme used in practice is variable-length. In the variable-length setting, if we use the code whose coding length is decided only from the empirical distribution (this code is called Lynch-Davisson code) in the i.i.d. case, the conditional distribution of the obtained data is the uniform distribution. That is, in the variable-length setting, there exists a code attaining the entropy rate with no error in both settings. Thus, a result different from the fixed-length setting can be expected in the the variable-length setting.

Furthermore, this type second order asymptotics can be extended to other topics in information theory. Indeed, in the case of channel coding, resolvability, and simple hypothesis 
testing, lemmas corresponding to Lemmas 144 have been obtained by Han [4]. Thus, it is not difficult to derive theorems corresponding to Theorem 3 However, in channel coding it is difficult to calculate the quantities corresponding to $\underline{H}(\epsilon, a \mid \bar{p})$ and $\bar{H}(\epsilon, a \mid \bar{p})$ even in the i.i.d. case. On the other hand, similar to fixed-length source coding and intrinsic randomness, we can treat the second order asymptotics concerning the other two problems in the i.i.d. case. Especially, when we discuss simple hypothesis testing with hypothesis $p$ and $q$ from the second order asymptotics viewpoint, we optimize the second order coefficient $b$ of the first error $e^{-n D(p \| q)-\sqrt{n} b}$ under the constraint that the second error probability is less than the fixed constant $\epsilon$. There is no difficulty in this problem. However, there is considerable difficulty in the quantum setting of this problem.

In addition, third order asymptotics is expected, but it seems difficult. In this extension of the i.i.d. case, our issue is the difference of $\sqrt{n}\left(-\frac{1}{n} \log P^{n}-H(P)\right)$ from the normal distribution. If the next order is a constant term of $\log P^{n}$, we cannot use methods similar to those described in this paper. This is an interesting future problem.

\section{ACKNOWLEDGMENTS}

The author would like to thank Professor Hiroshi Imai of the QCI project for support. He is grateful to Mr. Tsuyoshi Ito and Dr. Mitsuru Hamada for useful discussions. He also appreciates reviewers' helpful comments.

\section{APPENDIX}

Proof of 47 $\Rightarrow$ 48: $\quad$ The relations

$$
\begin{aligned}
& D\left(p_{n} \circ \phi_{n}^{-1} \| p_{U, \mathcal{M}_{n}}\right)=\log M_{n}-H\left(p_{n} \circ \phi_{n}^{-1}\right) \\
= & H\left(p_{U, \mathcal{M}_{n}}\right)-H\left(p_{n} \circ \phi_{n}^{-1}\right)
\end{aligned}
$$

hold.

If $d\left(p_{n} \circ \phi_{n}^{-1}, p_{U, \mathcal{M}_{n}}\right) \leq 1 / 4$, Fannes' inequality [16] (See also Csiszár and Körner [14]) implies

$$
\begin{aligned}
& \left|H\left(p_{U, \mathcal{M}_{n}}\right)-H\left(p_{n} \circ \phi_{n}^{-1}\right)\right| \\
\leq & -d\left(p_{n} \circ \phi_{n}^{-1}, p_{U, \mathcal{M}_{n}}\right) \log \left(d\left(p_{n} \circ \phi_{n}^{-1}, p_{U, \mathcal{M}_{n}}\right) / M_{n}\right) .
\end{aligned}
$$

Dividing the above by $n$, we have

$$
\begin{aligned}
& \frac{1}{n} D\left(p_{n} \circ \phi_{n}^{-1} \| p_{U, \mathcal{M}_{n}}\right) \\
\leq & d\left(p_{n} \circ \phi_{n}^{-1}, p_{U, \mathcal{M}_{n}}\right) \frac{1}{n}\left(\log M_{n}-\log \left(d\left(p_{n} \circ \phi_{n}^{-1}, p_{U, \mathcal{M}_{n}}\right)\right) .\right.
\end{aligned}
$$

Since $\varlimsup \frac{1}{n} \log M_{n}<\infty$, we obtain 47. 48 .

\section{REFERENCES}

[1] T.-S. Han, "Information-Spectrum Approaches: Tutorial," IEICE Technical Report, IT 2003-54, 31-39 (2003).

[2] I. Kontoyiannis, "Second-order noiseless source coding theorems," IEEE Trans. Inform. Theory, 43, 1339-1341 (1997).

[3] B. S. Clarke and A. R. Barron, "Jeffreys' prior is asymptotically least favorable under entropy risk," Journal of Statistical Planning and Inference, 41, 37-61 (1994).

[4] T.-S. Han, Information-Spectrum Methods in Information Theory, (Springer, Berlin, 2003). (Originally published by Baifukan 1998 in Japanese)
[5] T.-S. Han, "Folklore in Source coding: Information-Spectrum Approach," IEEE Trans. Inform. Theory, 51, 747-753 (2004).

[6] K. Visweswariah, S. Kulkarni and S. Verdú, "Source codes as random number generators," IEEE Trans. Inform. Theory, 44, 462-471 (1998).

[7] T.-S. Han and O. Uchida, "Source code with cost as a nonuniform random number generator," IEEE Trans. Inform. Theory, 46, 712-717 (2000).

[8] Y. Steinberg and S. Verdú, "Simulation of random processes and ratedistortion theory," IEEE Trans. Inform. Theory, 42, 63-86 (1996).

[9] P.-N. Chen and F. Alajaji, "Optimistic Shannon Coding Theorems for Arbitrary Single-User Systems," IEEE Trans. Inform. Theory, 45, 26232629 (1999).

[10] H. Nagaoka and M. Hayashi, "An information-spectrum approach to classical and quantum hypothesis testing," e-print quant-ph/0206185 (2002). [Online]. Available: http://arxiv.org/abs/quant-ph/0206185

[11] S. Vembu and S. Verdú, "Generating random bits from an arbitrary source: fundamental limits," IEEE Trans. Inform. Theory, 41, 1322-1332 (1995).

[12] M. Hayashi, "General formulas for fixed-length quantum entanglement concentration," IEEE Trans. Inform. Theory, 52, 1904-1921 (2006).

[13] A. Dembo and O. Zeitouni, Large deviation Techniques and Applications, (Springer, Berlin Heidelberg New York, 1997).

[14] I. Csiszár and J. Körner, Information Theory: Coding Theorems for Discrete Memoryless Systems, (Academic Press, New York, 1981).

[15] Y. Oohama and H. Sugano, "Adaptive Random Number Generation Using Arithmetic Coding and Its Performance Analysis," IEICE Technical Report, IT 98-60, 31-36 (1999), (In Japanese).

[16] M. Fannes, "A continuity property of the entropy density for spin lattice systems," Comm. Math. Phys., 31, 291-294 (1973). 